\title{
Evaluating the Role of Fertilizer and Seed Soaking on Direct Seeded Aus Rice Varieties
}

\section{Sushan Chowhan* and Kamrun Nahar}

Bangladesh Institute of Nuclear Agriculture (BINA), Bangladesh

*Corresponding Author: Sushan Chowhan, Adaptive Research and Extension

Division, Bangladesh Institute of Nuclear Agriculture (BINA), Bangladesh.
Received: December 14, 2021

Published: January 12, 2022

(C) All rights are reserved by Sushan Chowhan and Kamrun Nahar.

\begin{abstract}
Using conservation agriculture strategies is time demanding as intensive crop cultivation has already been started to feed the ever-growing population. Rice requires extensive resources for production. To preserve agroecosystem and sustain rice production it is imperative to consider soil and environmental health. Direct seeded rice (DSR) is a promising technology for rice cultivation which needs less input and resources. Aus season of Bangladesh is highly favorable for DSR cultivation. Being a new technology, farmers' lack enough information and knowledge on production technology of direct seeded aus rice. Taking into account, the present investigation was implemented to assess the response of some modern aus varieties, fertilizer doses and seed soaking methods on the yield attributes under DSR. The experiment was laid in factorial RCBD. Three factors considered in the experiment were- variety, fertilizer dose and seed soaking. Rice varieties chosen were- Binadhan-19 $\left(\mathrm{V}_{1}\right)$, Binadhan-21 $\left(\mathrm{V}_{2}\right)$, BRRI dhan82 $\left(\mathrm{V}_{3}\right)$ and BRRI dhan85 ( $\left.\mathrm{V}_{4}\right)$. Fertilizer doses (\% of recommended) were- 80\% NPKSZn ( $\left.\mathrm{T}_{1}\right), 100 \%$ NPKSZn $\left(\mathrm{T}_{2}\right)$ and $120 \%$ NPKSZn ( $\mathrm{T}_{3}$ ). Seed soaking methods were dry seeds directly sown without any soaking $\left(\mathrm{S}_{0}\right)$ and seeds sown after hydro soaking for $24 \mathrm{hrs}\left(\mathrm{S}_{24}\right)$. Data on yield and morpho physical attributes were collected randomly from five hills at final harvest. Outcomes revealed, longest panicle in $V_{3} \times T_{1} \times S_{24}(27.14$ $\mathrm{cm}$ ), treatment $\mathrm{V}_{4} \times \mathrm{T}_{1} \times \mathrm{S}_{0}$ had most filled grains (236.96) and $\mathrm{V}_{4} \times \mathrm{T}_{2} \times \mathrm{S}_{0}$ had least amount of filled grains (101.24) per panicle. Highest grain sterility was observed with $\mathrm{V}_{2} \times \mathrm{T}_{3} \times \mathrm{S}_{0}(48.17 \%)$ and the lowest with treatment $\mathrm{V}_{4} \times \mathrm{T}_{1} \times \mathrm{S}_{0}(27.40 \%)$. Lightest thousand grain weight (TGW) was found in $\mathrm{V}_{2} \times \mathrm{T}_{2} \times \mathrm{S}_{24}(19.59 \mathrm{~g})$; whereas, it was $17.45 \%$ more in treatment $\mathrm{V}_{1} \times \mathrm{T}_{2} \times \mathrm{S}_{24}(23.73 \mathrm{~g})$. Grain yield was maximum with treatment combination $\mathrm{V}_{4} \times \mathrm{T}_{1} \times \mathrm{S}_{0}(4.78 \mathrm{t} / \mathrm{ha})$ followed by a 7.17\% reduction in $\mathrm{V}_{2} \times \mathrm{T}_{3} \times \mathrm{S}_{0}(4.46 \mathrm{t} / \mathrm{ha})$ and $64 \%$ decrease with $\mathrm{V}_{4} \times \mathrm{T}_{3} \times \mathrm{S}_{24}(1.72 \mathrm{t} / \mathrm{ha})$. Late harvesting was seen in $\mathrm{V}_{4} \times \mathrm{T}_{3} \times \mathrm{S}_{24}(112.67$ days $)$; while $\mathrm{V}_{1} \times \mathrm{T}_{2} \times \mathrm{S}_{0}$ was the earliest (95.00 days). Above findings imply that, BRRI dhan85 and Binadhan-21 might be better with yields in dry-DSR without soaking; but balanced use of fertilizers should be ensured in order to avoid higher grain sterility and yield reduction.
\end{abstract}

Keywords: Direct Seeded Rice; Aus; Binadhan; Seed Soaking; Hydro Soaking; Fertilizer Management

\section{Introduction}

Geographical, soil and climatic conditions of Bangladesh permits year-round production of rice. More than half of the country's total population lives in rural area permitting involvement in agriculture and crop related activities. Rice is the common produce in the village area and the market economics is also greatly influenced by this crop. Rice is grown thrice a year in Bangladesh depending on area, land type and season; among them aus rice is (one of the rice growing season) cultivated during the dry period (kharif) of the year (March to June) which is mostly rain dependent and requires less fertilizer and irrigation. Though, aus rice needs less input but its cultivation is low in terms of land area due to low yield, more pest infestation, unavailability irrigation water [1], long drought periods etc. Besides, market price of rice is very unstable which often creates a scope for less profit to the growers. Earning more profit and reducing production related cost is impossible without applying new technologies of modern crops cultivation [2].

Direct seeded rice (DSR) cultivation is the latest and cost saving technology for aus rice cultivation. It's the way of broadcasted sowing or drilling or dibbling of dry or soaked (pregerminated) seeds directly in to the dry or wet or standing water soil surface $[3,4]$. There are three types of direct seeding system i.e. dry DSR, wet DSR and water seeding [5]. Conventional tillage transplanted rice (CT-TPR) requires more water (up to $40 \%$ of the total water for crop production) to prepare land, many labors (25 people ha-1 day $^{-1}$ ), high amount of fertilizers, pesticides, and have negative 
effects on soil and environment [6,7]. DSR is a type of conservation agriculture that ensures minimized disturbance of soil, flora, fauna, ecosystem, favors crop rotation and soil cover through crop residues [8]. Additionally, this type of cultivation ensures judicial water use, aids crops against drought, emits less methane [9], prevents plough pan formation in sub-soil [10], easy and time saving, reduces crop duration [11], conducive to mechanization [12] and also increase yield than CT-TPR [13], Besides, farmers can save $56 \%$ human labour, $73 \%$ machine labour and $25 \%$ irrigation water than CT-TPR; thus, DSR can enhance economic earning and save natural resources [14]. TPR and DSR yield increase against DRR (drum seeded rice) were 21.1 and 16.8 percent, respectively. But maximum net return and return per rupee financed were obtained by DSR. [15]. On the other hand, system of rice intensification (SRI) method has higher yield, less seed requirement for seedling and moderate water savings (50\%) but it is mostly adapted for rainfed rice as well as wheat, sugarcane and teff crops [16]. SRI was highly successful rice establishment technique in terms of nutrient absorption, usage efficiency and improvement of soil chemical and microbiological properties [17]. Despite many advantages of SRI; farmers preferred DSR due to less labor requirement, minimal special care, management and low costing. Plot selection and preparation, good crop establishment, precise weed, water, pest and fertilizer management are the key prerequisites for successful DSR [6]. Development of short duration, high yielding, water saving, low fertilizer requiring modern aus rice varieties along with different integrated approaches of mechanization, pest management and finally cost saving techniques has drawn farmers' interest towards adoption of DSR now a days [18]. During 2019, national total land area under rice production was 1,15,16,553 ha and average milled equivalent yield was $3.16 \mathrm{t} / \mathrm{ha}$ [19]. Whereas, the total aus production was limited to 27,06,320 ha during 2019-20 [20]. In 2020 Pab- na district, produced 29,870 tonnes aus rice from a land of 11,910 ha bearing a mean yield of $2.51 \mathrm{t} / \mathrm{ha}$. So, it's clear that yield of aus rice is much lower than the national average rice yield. Ishurdi upazila has limited aus coverage where suitable land area is about 2,083 ha [21]. Among the aus grown rice varieties approximately $85 \%$ are modern and $15 \%$ are traditional [22].

Yield in DSR is generally less than TPR because of poor crop establishment, higher grain sterility and pest infestation [23]. Moreover, due to imbalanced macro and micro nutrient fertilization management as well as high infiltration rates in DSR reduces overall yield [24]. Therefore, priming (soaking in water) is a major operation in DSR. As seeds are sowed directly, if the seeds are soaked in water up to a certain time it might assist in rapid germination and crop establishment in the field. Presently aus farmers do not apply all the recommended agronomic management during DSR cultivation thus they face enormous problems and that ultimately results in poor yield of the modern varieties. Taking these problem(s) in mind the present investigation was implemented to unveil the role of different fertilizer dose with priming application in the seeds of modern aus rice varieties and to suggest appropriate management strategy to the aus grower community.

\section{Materials and Methods}

\section{Experimental site}

This trail was conducted during the kharif season of 2020 at BINA Sub-station farm, Ishurdi, Pabna. It was under Agro Ecological Zone (AEZ) 10. Soil of this region is moderately fertile containing low amounts of organic matter having higher cation exchange capacity and lack in N, P, K, B and Zn [25]. An overview of different weather parameters throughout the experiment period is shown in figure 1 and figure 2 .

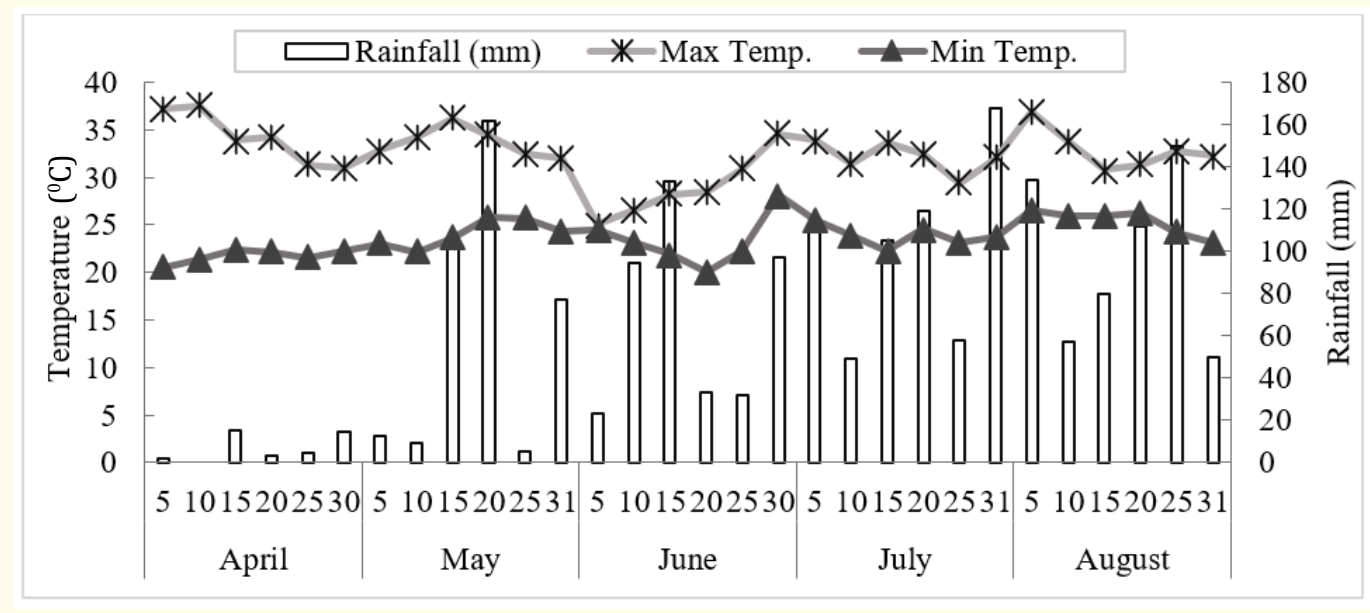

Figure 1: Rainfall and Temperature status during the study period of 2020. (Source: PRC [26]). 


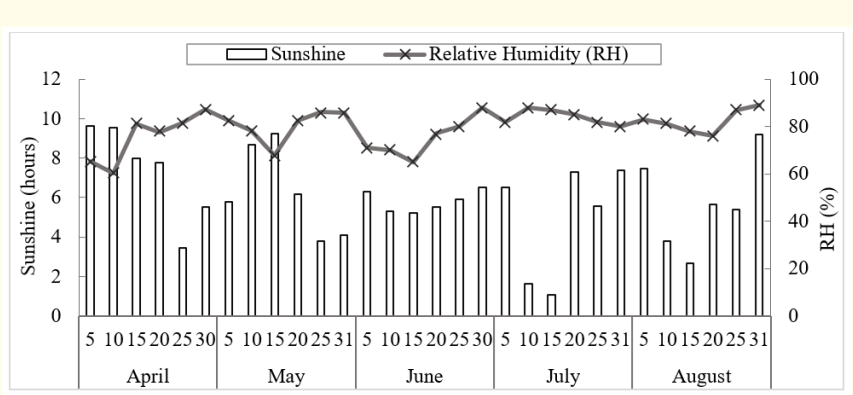

Figure 2: Sunshine and Relative humidity data of the trial site at 2020. (Source: PRC [26]).

\section{Crop management}

Experimental plot was tilled with power tiller to make the soil loose enough. Debris and weeds were collected and cleaned from the whole plots. Later the plot was levelled with ladder and individual treatment plots were prepared which consisted a size of $2 \mathrm{~m}$ $\times 2 \mathrm{~m}$ i.e. $4 \mathrm{~m}^{2}$ area of a unit plot. As this was a dry DSR experiment thus, seeds were line broadcasted in joe (containing enough soil moisture to germinate seeds) condition. Recommended fertilizer dose $(\mathrm{Kg} / \mathrm{ha})$ was $\mathrm{N}=96, \mathrm{P}=12, \mathrm{~K}=60, \mathrm{~S}=9$ and $\mathrm{Zn}=1.40$ respectively. Full dose of TSP, Gypsum, Zinc, $1 / 3^{\text {rd }}$ of MoP was applied as basal dose followed by harrowing. Later $1 / 3^{\text {rd }}$ of urea, $1 / 3^{\text {rd }}$ of MoP was applied at 10-12 DAE (days after emergence). Rest $1 / 3^{\text {rd }}$ of urea and MoP at 25-26 DAE and remaining urea was applied at 36-38 DAE [27,28]. $40 \mathrm{~g}$ seeds $(100 \mathrm{~kg} / \mathrm{ha})$ were line broadcasted in each plot $\left(4 \mathrm{~m}^{2}\right)$. After completion of sowing and experiment set, pre-emergence weedicide "Pendimethalin" (Trade name: Nirani plus 33 EC; East west chemicals ltd.) was sprayed at a rate of $3 \mathrm{ml} / \mathrm{L}$ [29] on next day i.e. 2 DAS (days after sowing). Few hours after spray a light irrigation was given on the next day so that soil does not dries completely. At 20 DAS, Bispyribac sodium (Prune 20WP, Auto Crop Care Limited) + Ethoxysulfuron (Sunrise 150 WG, Bayer CropScience Limited) was combinedly $(20 \mathrm{~g}+10 \mathrm{~g})$ sprayed at a rate of $1.5 \mathrm{~g} / \mathrm{L}[30,31]$. To prevent yellow stem borer infestation Virtako 40 WG (Syngenta Bangladesh Limited) was applied with during the 2 nd and $3^{\text {rd }}$ dose of urea application as per the procedure followed by Chowhan., et al. [32]. Hand weeding was done two times (35 DAS and 60 DAS) as per the methods described by Ahmed., et al. [33], Ahmed and Chauhan [34]. An outline of the experimental plot(s) is displayed in figure 3 and figure 4 .

Experimental design

A factorial Randomized complete block design (RCBD) with three replicates was followed to set the experiment. Details of the factors and treatments are mentioned below-

Factor (A): Variety (4)-

$\mathrm{V}_{1}=$ Binadhan $-19, \mathrm{~V}_{2}=$ Binadhan $-21, \mathrm{~V}_{3}=\mathrm{BRRI}$ dhan82, $\mathrm{V}_{4}=\mathrm{BRRI}$ dhan85

Factor (B): Fertilizer dose (3) (Kg/ha)-

$\mathrm{T}_{1}=80 \%$ recommended NPKSZn (Urea 167.2, TSP 47.432, MoP 64.26, Gypsum 26.66, Zn 3.10)

$\mathrm{T}_{2}=100 \%$ recommended NPKSZn (Urea 209, TSP 59.29, MoP 80.32, Gypsum 33.33, Zn 3.88)

$\mathrm{T}_{3}=120 \%$ recommended NPKSZn (Urea 250.8, TSP 71.15, MoP 96.38, Gypsum- 40, Zn- 4.66).

Factor (C): Seed soaking (2)-

$\mathrm{S}_{0}=$ Dry seeds (without soaking), $\mathrm{S}_{24}=$ Soaking for $24 \mathrm{hrs}$.

There were 72 treatment combinations thus comprising total 72 unit plots in three replicates.

\section{Observations and analysis}

Data on morpho physical and yield attributes were collected at final harvest. Randomly plants were selected from 5 hills and the following data were recorded on- Days to $50 \%$ emergence, Days to $90 \%$ emergence, Plant population per unit plot $\left(4 \mathrm{~m}^{2}\right)$, Days to $1^{\text {st }}$ flowering, Days to $50 \%$ flowering, Days to $90 \%$ flowering, Plant height (cm), Number of tillers/hill, Number of non-effective tillers/ hill, Number of panicles/hill, Panicle length $(\mathrm{cm})$, Panicle weight (g), Root length $\left(\mathrm{cm}^{2}\right)$, Number of filled grains/panicle, Unfilled grains/panicle (\%), 1000 grain weight (TGW) (g), Grain yield (t/ ha), Straw yield ( $\mathrm{t} / \mathrm{ha}$ ) and Days to harvest. Plant population were recorded through hand counting and flowering data were collected from eye observation. Grain yield was adjusted at $14 \%$ moisture content to convert the total yield ( $\mathrm{t} / \mathrm{ha}$ ). After harvesting the total unit plot straws were sundried till it's color turned yellow and then weighed to calculate the straw yield ( $\mathrm{t} / \mathrm{ha}$ ). After that, all gathered data were statistically separately analyzed with ANOVA (analysis of variance) technique through Statistix 10 software [35]. Significance of mean difference was compared by LSD (least significant difference) test $[36,37]$ at $5 \%$ level of probability. 


\begin{tabular}{|c|c|c|c|c|c|c|c|c|}
\hline \multicolumn{3}{|c|}{$\mathbf{R}_{1}$} & \multicolumn{3}{|c|}{$\mathbf{R}_{\mathbf{2}}$} & \multicolumn{3}{|c|}{$\mathbf{R}_{3}$} \\
\hline \multicolumn{9}{|c|}{$\longleftrightarrow 1 \mathrm{~m}$} \\
\hline $\mathrm{V}_{1} \mathrm{~T}_{1} \mathrm{~S}_{0}$ & $\mathrm{~V}_{2} \mathrm{~T}_{2} \mathrm{~S}_{0}$ & $\mathrm{~V}_{3} \mathrm{~T}_{3} \mathrm{~S}_{0}$ & & $\begin{array}{l}\mathrm{V}_{2} \mathrm{~T}_{2} \mathrm{~S}_{0} \\
0.5 \mathrm{~m}\end{array}$ & $\mathrm{~V}_{3} \mathrm{~T}_{3} \mathrm{~S}_{0}$ & $\mathrm{~V}_{1} \mathrm{~T}_{1} \mathrm{~S}_{0}$ & $\mathrm{~V}_{2} \mathrm{~T}_{2} \mathrm{~S}_{0}$ & $\mathrm{~V}_{3} \mathrm{~T}_{3} \mathrm{~S}_{0}$ \\
\hline $\mathrm{V}_{1} \mathrm{~T}_{1} \mathrm{~S}_{24}$ & $\mathrm{~V}_{2} \mathrm{~T}_{2} \mathrm{~S}_{24}$ & $\mathrm{~V}_{3} \mathrm{~T}_{3} \mathrm{~S}_{24}$ & $\mathrm{~V}_{1} \mathrm{~T}_{1} \mathrm{~S}_{24}$ & $\mathrm{~V}_{2} \mathrm{~T}_{2} \mathrm{~S}_{24}$ & $\mathrm{~V}_{3} \mathrm{~T}_{3} \mathrm{~S}_{24}$ & $\mathrm{~V}_{1} \mathrm{~T}_{1} \mathrm{~S}_{24}$ & $\mathrm{~V}_{2} \mathrm{~T}_{2} \mathrm{~S}_{24}$ & $\mathrm{~V}_{3} \mathrm{~T}_{3} \mathrm{~S}_{24}$ \\
\hline $\mathrm{V}_{1} \mathrm{~T}_{2} \mathrm{~S}_{0}$ & $\mathrm{~V}_{2} \mathrm{~T}_{3} \mathrm{~S}_{0}$ & $\mathrm{~V}_{4} \mathrm{~T}_{1} \mathrm{~S}_{0}$ & $\mathrm{~V}_{1} \mathrm{~T}_{2} \mathrm{~S}_{0}$ & $\mathrm{~V}_{2} \mathrm{~T}_{3} \mathrm{~S}_{0}$ & $\mathrm{~V}_{4} \mathrm{~T}_{1} \mathrm{~S}_{0}$ & $\mathrm{~V}_{1} \mathrm{~T}_{2} \mathrm{~S}_{0}$ & $\mathrm{~V}_{2} \mathrm{~T}_{3} \mathrm{~S}_{0}$ & $\mathrm{~V}_{4} \mathrm{~T}_{1} \mathrm{~S}_{0}$ \\
\hline $\mathrm{V}_{1} \mathrm{~T}_{2} \mathrm{~S}_{24}$ & $\mathrm{~V}_{2} \mathrm{~T}_{3} \mathrm{~S}_{24}$ & $\mathrm{~V}_{4} \mathrm{~T}_{1} \mathrm{~S}_{24}$ & $\mathrm{~V}_{1} \mathrm{~T}_{2} \mathrm{~S}_{24}$ & $\mathrm{~V}_{2} \mathrm{~T}_{3} \mathrm{~S}_{24}$ & $\mathrm{~V}_{4} \mathrm{~T}_{1} \mathrm{~S}_{24}$ & $\mathrm{~V}_{1} \mathrm{~T}_{2} \mathrm{~S}_{24}$ & $\mathrm{~V}_{2} \mathrm{~T}_{3} \mathrm{~S}_{24}$ & $\mathrm{~V}_{4} \mathrm{~T}_{1} \mathrm{~S}_{24}$ \\
\hline $\mathrm{V}_{1} \mathrm{~T}_{3} \mathrm{~S}_{0}$ & $\mathrm{~V}_{3} \mathrm{~T}_{1} \mathrm{~S}_{0}$ & $\mathrm{~V}_{4} \mathrm{~T}_{2} \mathrm{~S}_{0}$ & $\mathrm{~V}_{1} \mathrm{~T}_{3} \mathrm{~S}_{0}$ & $\mathrm{~V}_{3} \mathrm{~T}_{1} \mathrm{~S}_{0}$ & $\mathrm{~V}_{4} \mathrm{~T}_{2} \mathrm{~S}_{0}$ & $\mathrm{~V}_{1} \mathrm{~T}_{3} \mathrm{~S}_{0}$ & $\mathrm{~V}_{3} \mathrm{~T}_{1} \mathrm{~S}_{0}$ & $\mathrm{~V}_{4} \mathrm{~T}_{2} \mathrm{~S}_{0}$ \\
\hline $\mathrm{V}_{1} \mathrm{~T}_{3} \mathrm{~S}_{24}$ & $\mathrm{~V}_{3} \mathrm{~T}_{1} \mathrm{~S}_{24}$ & $\mathrm{~V}_{4} \mathrm{~T}_{2} \mathrm{~S}_{24}$ & $\mathrm{~V}_{1} \mathrm{~T}_{3} \mathrm{~S}_{24}$ & $\mathrm{~V}_{3} \mathrm{~T}_{1} \mathrm{~S}_{24}$ & $\mathrm{~V}_{4} \mathrm{~T}_{2} \mathrm{~S}_{24}$ & $\mathrm{~V}_{1} \mathrm{~T}_{3} \mathrm{~S}_{24}$ & $\mathrm{~V}_{3} \mathrm{~T}_{1} \mathrm{~S}_{24}$ & $\mathrm{~V}_{4} \mathrm{~T}_{2} \mathrm{~S}_{24}$ \\
\hline $\mathrm{V}_{2} \mathrm{~T}_{1} \mathrm{~S}_{0}$ & $\mathrm{~V}_{3} \mathrm{~T}_{2} \mathrm{~S}_{0}$ & $\mathrm{~V}_{4} \mathrm{~T}_{3} \mathrm{~S}_{0}$ & $\mathrm{~V}_{2} \mathrm{~T}_{1} \mathrm{~S}_{0}$ & $\mathrm{~V}_{3} \mathrm{~T}_{2} \mathrm{~S}_{0}$ & $\mathrm{~V}_{4} \mathrm{~T}_{3} \mathrm{~S}_{0}$ & $\mathrm{~V}_{2} \mathrm{~T}_{1} \mathrm{~S}_{0}$ & $\mathrm{~V}_{3} \mathrm{~T}_{2} \mathrm{~S}_{0}$ & $\mathrm{~V}_{4} \mathrm{~T}_{3} \mathrm{~S}_{0}$ \\
\hline $\mathrm{V}_{2} \mathrm{~T}_{1} \mathrm{~S}_{24}$ & $\mathrm{~V}_{3} \mathrm{~T}_{2} \mathrm{~S}_{24}$ & $\mathrm{~V}_{4} \mathrm{~T}_{3} \mathrm{~S}_{24}$ & $\mathrm{~V}_{2} \mathrm{~T}_{1} \mathrm{~S}_{24}$ & $\mathrm{~V}_{3} \mathrm{~T}_{2} \mathrm{~S}_{24}$ & $\mathrm{~V}_{4} \mathrm{~T}_{3} \mathrm{~S}_{24}$ & $\mathrm{~V}_{2} \mathrm{~T}_{1} \mathrm{~S}_{24}$ & $\mathrm{~V}_{3} \mathrm{~T}_{2} \mathrm{~S}_{24}$ & $\mathrm{~V}_{4} \mathrm{~T}_{3} \mathrm{~S}_{24}$ \\
\hline
\end{tabular}

Figure 3: Field layout of the experiment.
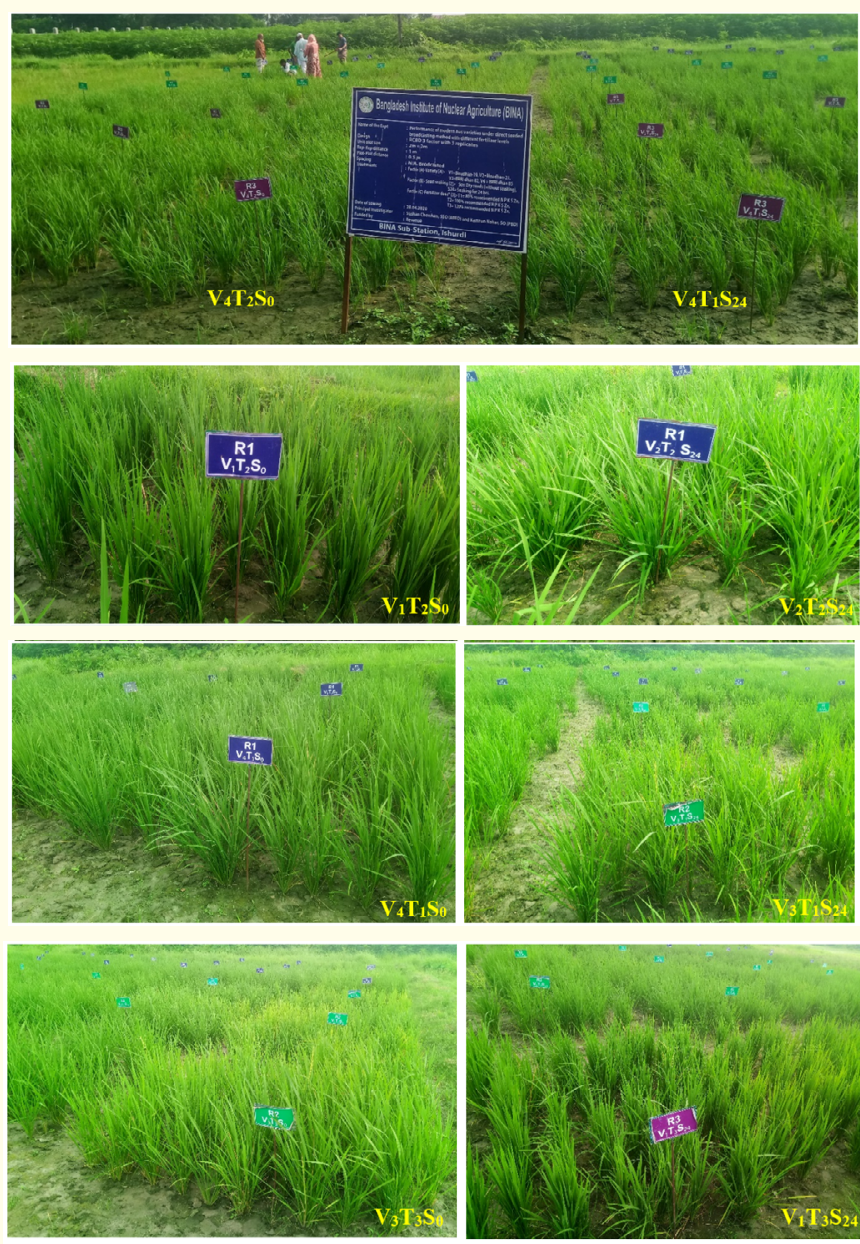

Figure 4: Field view of the DSR experiment with individual treatment (yellow lettered) plots at vegetative stage.

\section{Results and Discussion}

\section{Days to emergence}

Significant deviations were observed in terms of seedling emergence within various treatments (Table 1 and Table 2). With varietal and fertilizer effect, maximum days to $50 \%$ (13.5 days) and $90 \%$ (18.67 days) emergence was noted with treatment $\mathrm{V}_{4} \times \mathrm{T}_{3}$. Contrary, least days to $50 \%$ (9.33 days) and 90\% (14.33 days) emergence was seen with the treatments $V_{2} \times T_{2}$ and $V_{1} \times T_{1}$.

Effect of variety and soaking methods exhibited that, treatment $\mathrm{V}_{4} \times \mathrm{S}_{24}$ took most days to $50 \%$ (12.11 days) and $90 \%$ (17.67 days) emergence. Whereas, earliest emergence (50\% and 90\%) was noticed with treatment $\mathrm{V}_{1} \times \mathrm{S}_{0}(9.78$ days and 14.44 days $)$.

In case of fertilizer dose and soaking method impact, highest days to $50 \%$ (12.17 days) and 90\% (17.42 days) emergence was spotted with $\mathrm{T}_{3} \times \mathrm{S}_{24}$. While, treatment $\mathrm{T}_{2} \times \mathrm{S}_{0}$ emerged in advance in case of $50 \%$ (10.00 days) and $90 \%$ (14.92 days).

Interaction effect of variety, fertilizer and soaking method demonstrated that, $\mathrm{V}_{4} \times \mathrm{T}_{2} \times \mathrm{S}_{24}$ treatment had late emergence for $50 \%$ (14.00 days) and 90\% (20.67 days) than other treatment combinations. Conversely, earliest days to 50\% (8.33 days) and 90\% (13.00 days) emergence was sighted in treatment combination $\mathrm{V}_{2} \times \mathrm{T}_{2} \times \mathrm{S}_{0}$. Disparities in seedling emergence among the treatments might be due to uneven soil moisture, temperature, seed and varietal qualities. It was remarkably noticed that, seeds without soaked gained early emergence than the soaked ones. This occurred may be due to adequate soil moisture in the experimental plots. Yasumoto., et al. [38] found that rice seedling emergence was related with sowing time, season, temperature and cultivar type. 


\section{Plant population}

Desired plant density is an essential factor to ensure optimum growth and quality of the crop. Variety, fertilizer dose and soaking treatments indicated great variation in the number of plants (Table 1 and Table 2) per unit plot area $\left(4 \mathrm{~m}^{2}\right)$. Abundant plant population was observed with the treatment $V_{4} \times T_{1}$ (110.17) and scarce was noted in $\mathrm{V}_{1} \times \mathrm{T}_{3}$ (73.17) influenced by variety and fertilizer.

Influence variety and soaking disclosed that, $\mathrm{V}_{3} \times \mathrm{S}_{0}$ had largest plant population (109.67) and treatment $V_{1} \times S_{24}$ beard the scantiest number of plants (88.78) followed by $V_{4} \times S_{24}$ (88.89) which were statistically identical.

Fertilizer and soaking application effect denoted treatments $\mathrm{T}_{2} \times \mathrm{S}_{0}$ produced denser (104.70) plants but $\mathrm{T}_{3} \times \mathrm{S}_{24}$ had lighter (82.25) plant in the plot area.
Combined influences revealed that treatment combination $\mathrm{V}_{3} \times$ $\mathrm{T}_{2} \times \mathrm{S}_{0}$ gave utmost (123.33) plant population; while treatment $\mathrm{V}_{4}$ $\times \mathrm{T}_{3} \times \mathrm{S}_{24}$ had the lowest (55.67) population among the combinations. Plant population depended on the germination capability of the seeds as well as the soil moisture conditions. Seed rate might be a factor but all the plots got same amount of seed though plant density differed. These findings are more or less in conformity with Kaur and Singh [39] who concluded, number of plants per unit area is dependent on seed rate.

\section{Days to flowering}

Flower initiation were also remarkably varied among the treatments (Table 1 and Table 2). Days to $1^{\text {st }}, 50 \%$ and $90 \%$ flowering were observed late in the treatment $\mathrm{V}_{4} \times \mathrm{T}_{2}$ (76.17 days, 80.00 days and 84.17 days) and earlier in $\mathrm{V}_{3} \times \mathrm{T}_{2}$ (68.83 days, 73.67 days and 77.67 days) with variety and fertilizer effect.

\begin{tabular}{|c|c|c|c|c|c|c|}
\hline Treat. & $\begin{array}{l}\text { Days to } 50 \% \\
\text { emergence }\end{array}$ & $\begin{array}{c}\text { Days to } 90 \% \\
\text { emergence }\end{array}$ & $\begin{array}{c}\text { Plantpopulation@ } \\
4 \mathrm{~m}^{2}\end{array}$ & $\begin{array}{l}\text { Days to first } \\
\text { flowering }\end{array}$ & $\begin{array}{c}\text { Days to } 50 \% \\
\text { flowering }\end{array}$ & $\begin{array}{c}\text { Days to } 90 \% \\
\text { flowering }\end{array}$ \\
\hline \multicolumn{7}{|c|}{ Variety $\times$ Fertilizer dose } \\
\hline $\mathrm{V}_{1} \times \mathrm{T}_{1}$ & $10.00 \mathrm{~cd}$ & $14.33 \mathrm{c}$ & $107.83 \mathrm{ab}$ & $72.33 \mathrm{ef}$ & $76.17 \mathrm{de}$ & $80.17 \mathrm{~cd}$ \\
\hline $\mathrm{V}_{1} \times \mathrm{T}_{2}$ & $9.83 \mathrm{~d}$ & $15.50 \mathrm{c}$ & $101.33 \mathrm{ab}$ & $73.17 \mathrm{c}-\mathrm{f}$ & $76.50 \mathrm{~cd}$ & $81.17 \mathrm{bc}$ \\
\hline $\mathrm{V}_{1} \times \mathrm{T}_{3}$ & $10.50 \mathrm{bcd}$ & $15.33 \mathrm{c}$ & $73.17 \mathrm{c}$ & $73.50 \mathrm{~b}-\mathrm{e}$ & $76.83 \mathrm{bcd}$ & $80.83 \mathrm{c}$ \\
\hline $\mathrm{V}_{2} \times \mathrm{T}_{1}$ & $9.83 \mathrm{~d}$ & $14.50 \mathrm{c}$ & $85.17 \mathrm{abc}$ & $72.50 \mathrm{def}$ & $75.83 \mathrm{def}$ & $80.17 \mathrm{~cd}$ \\
\hline $\mathrm{V}_{2} \times \mathrm{T}_{2}$ & $9.33 \mathrm{~d}$ & $15.83 \mathrm{bc}$ & $103.17 \mathrm{ab}$ & 74.17 a-e & $77.17 \mathrm{bcd}$ & $81.00 \mathrm{c}$ \\
\hline $\mathrm{V}_{2} \times \mathrm{T}_{3}$ & $12.33 \mathrm{ab}$ & $16.33 \mathrm{abc}$ & $101.17 \mathrm{ab}$ & $75.00 \mathrm{a}-\mathrm{d}$ & $79.00 \mathrm{ab}$ & $82.00 \mathrm{abc}$ \\
\hline $\mathrm{V}_{3} \times \mathrm{T}_{1}$ & $11.17 \mathrm{bcd}$ & $15.50 \mathrm{c}$ & $94.17 \mathrm{abc}$ & $70.83 \mathrm{fg}$ & $75.67 \mathrm{def}$ & $78.17 \mathrm{~d}$ \\
\hline $\mathrm{V}_{3} \times \mathrm{T}_{2}$ & $10.67 \mathrm{bcd}$ & $16.67 \mathrm{abc}$ & $96.17 \mathrm{abc}$ & $68.83 \mathrm{~g}$ & $73.67 \mathrm{f}$ & $77.67 \mathrm{~d}$ \\
\hline $\mathrm{V}_{3} \times \mathrm{T}_{3}$ & $10.17 \mathrm{bcd}$ & $16.83 \mathrm{abc}$ & $108.50 \mathrm{ab}$ & $69.50 \mathrm{~g}$ & 73.83 ef & $78.17 \mathrm{~d}$ \\
\hline $\mathrm{V}_{4} \times \mathrm{T}_{1}$ & $9.17 \mathrm{~d}$ & $16.67 \mathrm{abc}$ & $110.17 \mathrm{a}$ & $76.00 \mathrm{ab}$ & $78.83 \mathrm{abc}$ & $83.83 \mathrm{a}$ \\
\hline $\mathrm{V}_{4} \times \mathrm{T}_{2}$ & $12.17 \mathrm{abc}$ & $18.33 \mathrm{ab}$ & $84.50 \mathrm{bc}$ & $76.17 \mathrm{a}$ & $80.00 \mathrm{a}$ & 84.17 a \\
\hline $\mathrm{V}_{4} \times \mathrm{T}_{3}$ & $13.50 \mathrm{a}$ & $18.67 \mathrm{a}$ & $73.83 \mathrm{c}$ & $75.17 \mathrm{abc}$ & $80.00 \mathrm{a}$ & $83.67 \mathrm{ab}$ \\
\hline LoS & $*$ & $*$ & $*$ & $*$ & $*$ & $*$ \\
\hline $\mathrm{LSD}_{0.05}$ & 2.33 & 2.71 & 25.53 & 2.53 & 2.49 & 2.51 \\
\hline SEm \pm & 1.16 & 1.35 & 12.68 & 1.26 & 1.24 & 1.25 \\
\hline \multicolumn{7}{|c|}{ Variety $\times$ Soaking method } \\
\hline $\mathrm{V}_{1} \times \mathrm{S}_{0}$ & $9.78 \mathrm{~b}$ & $14.44 \mathrm{~b}$ & $99.44 \mathrm{ab}$ & $72.22 \mathrm{~d}$ & $75.56 \mathrm{def}$ & $80.00 \mathrm{~cd}$ \\
\hline $\mathrm{V}_{1} \times \mathrm{S}_{24}$ & $10.44 \mathrm{ab}$ & $16.00 \mathrm{ab}$ & $88.78 \mathrm{~b}$ & $73.78 \mathrm{bcd}$ & $77.44 \mathrm{bcd}$ & 81.44 bc \\
\hline $\mathrm{V}_{2} \times \mathrm{S}_{0}$ & $10.22 \mathrm{ab}$ & $15.56 \mathrm{ab}$ & $96.67 \mathrm{ab}$ & $72.89 \mathrm{~cd}$ & 76.56 cde & $80.44 \mathrm{c}$ \\
\hline $\mathrm{V}_{2} \times \mathrm{S}_{24}$ & $10.78 \mathrm{ab}$ & $15.56 \mathrm{ab}$ & $96.33 \mathrm{ab}$ & $74.89 \mathrm{abc}$ & $78.11 \mathrm{abc}$ & $81.67 \mathrm{bc}$ \\
\hline $\mathrm{V}_{3} \times \mathrm{S}_{0}$ & $10.33 \mathrm{ab}$ & $15.78 \mathrm{ab}$ & $109.67 \mathrm{a}$ & $69.78 \mathrm{e}$ & $74.22 \mathrm{f}$ & $78.00 \mathrm{~d}$ \\
\hline $\mathrm{V}_{3} \times \mathrm{S}_{24}$ & $11.00 \mathrm{ab}$ & $17.33 \mathrm{a}$ & $89.56 \mathrm{ab}$ & $69.67 \mathrm{e}$ & 74.56 ef & $78.00 \mathrm{~d}$ \\
\hline $\mathrm{V}_{4} \times \mathrm{S}_{0}$ & $11.11 \mathrm{ab}$ & $17.33 \mathrm{a}$ & $92.11 \mathrm{ab}$ & $75.56 \mathrm{ab}$ & $79.11 \mathrm{ab}$ & $83.22 \mathrm{ab}$ \\
\hline $\mathrm{V}_{4} \times \mathrm{S}_{24}$ & $12.11 \mathrm{a}$ & $17.67 \mathrm{a}$ & $86.89 \mathrm{~b}$ & $76.00 \mathrm{a}$ & $80.11 \mathrm{a}$ & $84.56 \mathrm{a}$ \\
\hline $\mathrm{LSD}_{0.05}$ & 1.90 & 2.21 & 20.85 & 2.07 & 2.03 & 2.05 \\
\hline
\end{tabular}




\begin{tabular}{|c|c|c|c|c|c|c|}
\hline Treat. & $\begin{array}{c}\text { Days to } 50 \% \\
\text { emergence }\end{array}$ & $\begin{array}{c}\text { Days to } 90 \% \\
\text { emergence }\end{array}$ & $\begin{array}{c}\text { Plant population@ } \\
\mathbf{4 m}^{2}\end{array}$ & $\begin{array}{l}\text { Days to first } \\
\text { flowering }\end{array}$ & $\begin{array}{c}\text { Days to } 50 \% \\
\text { flowering }\end{array}$ & $\begin{array}{c}\text { Days to } 90 \% \\
\text { flowering }\end{array}$ \\
\hline LoS & * & * & * & $*$ & $*$ & $*$ \\
\hline SEm \pm & 0.94 & 1.10 & 10.36 & 1.03 & 1.01 & 1.02 \\
\hline \multicolumn{7}{|c|}{ Fertilizer dose $\times$ Soaking method } \\
\hline $\mathrm{T}_{1} \times \mathrm{S}_{0}$ & $10.00 \mathrm{~b}$ & $15.83 \mathrm{abc}$ & $98.17 \mathrm{ab}$ & $72.00 \mathrm{~b}$ & $75.67 \mathrm{~b}$ & $79.58 \mathrm{~b}$ \\
\hline $\mathrm{T}_{1} \times \mathrm{S}_{24}$ & $10.08 \mathrm{~b}$ & $15.50 \mathrm{bc}$ & $100.50 \mathrm{a}$ & 73.83 a & 77.58 a & 81.58 a \\
\hline $\mathrm{T}_{2} \times \mathrm{S}_{0}$ & $10.00 \mathrm{~b}$ & $14.92 \mathrm{c}$ & $104.17 \mathrm{a}$ & $72.50 \mathrm{ab}$ & $76.08 \mathrm{ab}$ & $80.42 \mathrm{ab}$ \\
\hline $\mathrm{T}_{2} \times \mathrm{S}_{24}$ & $11.00 \mathrm{ab}$ & $17.00 \mathrm{ab}$ & $88.42 \mathrm{ab}$ & $73.67 \mathrm{ab}$ & $77.58 \mathrm{a}$ & $81.58 \mathrm{a}$ \\
\hline $\mathrm{T}_{3} \times \mathrm{S}_{0}$ & $11.08 \mathrm{ab}$ & $16.58 \mathrm{abc}$ & $96.08 \mathrm{ab}$ & $73.33 \mathrm{ab}$ & $77.33 \mathrm{ab}$ & $81.25 \mathrm{ab}$ \\
\hline $\mathrm{T}_{3} \times \mathrm{S}_{24}$ & $12.17 \mathrm{a}$ & $17.42 \mathrm{a}$ & $82.25 \mathrm{~b}$ & $73.25 \mathrm{ab}$ & $77.50 \mathrm{a}$ & $81.08 \mathrm{ab}$ \\
\hline $\mathrm{LSD}_{0.05}$ & 1.65 & 1.91 & 18.05 & 1.79 & 1.76 & 1.77 \\
\hline LoS & $*$ & $*$ & $*$ & $*$ & $*$ & $*$ \\
\hline SEm \pm & 0.82 & 0.95 & 8.97 & 0.89 & 0.88 & 0.88 \\
\hline $\mathrm{CV}$ & $18.69 \%$ & $14.37 \%$ & $23.14 \%$ & $2.98 \%$ & $2.79 \%$ & $2.67 \%$ \\
\hline
\end{tabular}

Table 1: Phenology and ontogenetic features of aus rice with relation to variety, fertilizer dose and soaking.

Means bearing same letter(s) in a column do not differ significantly at 5\% level of probability by LSD. LoS: Level of Significance, SEm: Standard Error Mean, CV: Coefficient of Variation.

Varieties and soaking impact exposed that, $\mathrm{V}_{4} \times \mathrm{S}_{24}$ treatment took longest days to bear $1^{\text {st }}, 50 \%$ and $90 \%$ flowering (76.00 days, 80.11 days and 84.56 days) but, shortest days were seen with treatment $\mathrm{V}_{3} \times \mathrm{S}_{0}$ (69.78 days, 74.22 days and 78.00 days. Treatment $\mathrm{V}_{3}$ $\times \mathrm{S}_{24}$ also showed statistically identical days to $1^{\text {st }}$ (69.67 days) and 90\% (78.00 days) flowering.
Different doses of fertilizer and soaking revealed treatment $\mathrm{T}_{1}$ $\times \mathrm{S}_{24}$ gave late $1^{\text {st }}, 50 \%$ and $90 \%$ flowering (73.83 days, 77.58 days and 81.58 days). Therefore, days to $50 \%$ and $90 \%$ flowering were also delayed in treatments $\mathrm{T}_{2} \times \mathrm{S}_{24}$ (77.58 days and 81.58 days) followed by $\mathrm{T}_{3} \times \mathrm{S}_{24}$ (77.50 days and 81.08 days); all these were statistically identical.

\begin{tabular}{|c|c|c|c|c|c|c|}
\hline Treatment & $\begin{array}{c}\text { Days to } 50 \% \\
\text { emergence }\end{array}$ & $\begin{array}{c}\text { Days to } 90 \% \\
\text { emergence }\end{array}$ & $\begin{array}{c}\text { Plant population@ } \\
4 \mathbf{m}^{2}\end{array}$ & $\begin{array}{l}\text { Days to } 1^{\text {st }} \\
\text { flowering }\end{array}$ & $\begin{array}{c}\text { Days to } 50 \% \\
\text { flowering }\end{array}$ & $\begin{array}{c}\text { Days to } 90 \% \\
\text { flowering }\end{array}$ \\
\hline $\mathrm{V}_{1} \times \mathrm{T}_{1} \times \mathrm{S}_{0}$ & $9.00 \mathrm{ef}$ & $13.33 \mathrm{f}$ & $119.67 \mathrm{ab}$ & $70.67 \mathrm{~g}-\mathrm{k}$ & 74.33 ghi & 78.33 ef \\
\hline $\mathrm{V}_{1} \times \mathrm{T}_{2} \times \mathrm{S}_{0}$ & $11.00 \mathrm{a}-\mathrm{f}$ & $15.67 \mathrm{c}-\mathrm{f}$ & 107.33 a-e & $72.33 \mathrm{~d}-\mathrm{j}$ & $76.00 \mathrm{~d}-\mathrm{i}$ & $81.00 \mathrm{~b}-\mathrm{f}$ \\
\hline $\mathrm{V}_{1} \times \mathrm{T}_{3} \times \mathrm{S}_{0}$ & $9.33 \mathrm{def}$ & 14.33 ef & $71.33 \mathrm{efg}$ & $73.67 \mathrm{a}-\mathrm{g}$ & $76.33 \mathrm{~d}-\mathrm{i}$ & $80.67 \mathrm{c}-\mathrm{f}$ \\
\hline $\mathrm{V}_{2} \times \mathrm{T}_{1} \times \mathrm{S}_{0}$ & $10.00 \mathrm{def}$ & $16.00 \mathrm{~b}-\mathrm{f}$ & $79.00 \mathrm{c}-\mathrm{g}$ & 71.33 e-k & $75.00 \mathrm{f}-\mathrm{i}$ & $79.33 \mathrm{def}$ \\
\hline $\mathrm{V}_{2} \times \mathrm{T}_{2} \times \mathrm{S}_{0}$ & $8.33 \mathrm{f}$ & $13.00 \mathrm{f}$ & 102.00 a-f & $72.67 \mathrm{c}-\mathrm{i}$ & $75.67 \mathrm{e}-\mathrm{i}$ & $80.00 \mathrm{c}-\mathrm{f}$ \\
\hline $\mathrm{V}_{2} \times \mathrm{T}_{3} \times \mathrm{S}_{0}$ & $12.33 \mathrm{a}-\mathrm{d}$ & 17.67 a-e & $109.00 \mathrm{a}-\mathrm{d}$ & 74.67 a-e & 79.00 a-e & $82.00 \mathrm{a}-\mathrm{d}$ \\
\hline $\mathrm{V}_{3} \times \mathrm{T}_{1} \times \mathrm{S}_{0}$ & $11.67 \mathrm{a}-\mathrm{e}$ & 17.67 a-e & $93.67 \mathrm{a}-\mathrm{f}$ & $71.00 \mathrm{f}-\mathrm{k}$ & $75.67 \mathrm{e}-\mathrm{i}$ & 78.33 ef \\
\hline $\mathrm{V}_{3} \times \mathrm{T}_{2} \times \mathrm{S}_{0}$ & $10.33 \mathrm{c}-\mathrm{f}$ & $15.00 \mathrm{c}-\mathrm{f}$ & $123.33 \mathrm{a}$ & $69.33 \mathrm{ijk}$ & 73.67 hi & $77.67 \mathrm{f}$ \\
\hline $\mathrm{V}_{3} \times \mathrm{T}_{3} \times \mathrm{S}_{0}$ & 9.00 ef & $14.67 \mathrm{def}$ & $112.00 \mathrm{abc}$ & $69.00 \mathrm{jk}$ & $73.33 \mathrm{i}$ & 78.00 ef \\
\hline $\mathrm{V}_{4} \times \mathrm{T}_{1} \times \mathrm{S}_{0}$ & $9.33 \mathrm{def}$ & $16.33 \mathrm{~b}-\mathrm{f}$ & $100.33 \mathrm{a}-\mathrm{f}$ & $75.00 \mathrm{a}-\mathrm{d}$ & $77.67 \mathrm{a}-\mathrm{g}$ & $82.33 \mathrm{a}-\mathrm{d}$ \\
\hline $\mathrm{V}_{4} \times \mathrm{T}_{2} \times \mathrm{S}_{0}$ & $10.33 \mathrm{c}-\mathrm{f}$ & $16.00 \mathrm{~b}-\mathrm{f}$ & $84.00 \mathrm{~b}-\mathrm{g}$ & $75.67 \mathrm{a}-\mathrm{d}$ & 79.00 a-e & $83.00 \mathrm{abc}$ \\
\hline $\mathrm{V}_{4} \times \mathrm{T}_{3} \times \mathrm{S}_{0}$ & $13.67 \mathrm{ab}$ & $19.67 \mathrm{ab}$ & $92.00 \mathrm{a}-\mathrm{f}$ & $76.00 \mathrm{abc}$ & $80.67 \mathrm{ab}$ & $84.33 \mathrm{ab}$ \\
\hline $\mathrm{V}_{1} \times \mathrm{T}_{1} \times \mathrm{S}_{24}$ & $11.00 \mathrm{a}-\mathrm{f}$ & 17.67 a-e & $96.00 \mathrm{a}-\mathrm{f}$ & $74.00 \mathrm{a}-\mathrm{g}$ & 78.00 a-f & $82.00 \mathrm{a}-\mathrm{d}$ \\
\hline $\mathrm{V}_{1} \times \mathrm{T}_{2} \times \mathrm{S}_{24}$ & 8.67 ef & $13.96 \mathrm{f}$ & 95.33 a-f & $74.00 \mathrm{a}-\mathrm{g}$ & $77.00 \mathrm{c}-\mathrm{h}$ & $81.33 \mathrm{~b}-\mathrm{e}$ \\
\hline $\mathrm{V}_{1} \times \mathrm{T}_{3} \times \mathrm{S}_{24}$ & 11.67 a-e & 17.33 a-e & $75.00 \mathrm{~d}-\mathrm{g}$ & $73.33 \mathrm{~b}-\mathrm{h}$ & $77.33 \mathrm{~b}-\mathrm{g}$ & $81.00 \mathrm{~b}-\mathrm{f}$ \\
\hline $\mathrm{V}_{2} \times \mathrm{T}_{1} \times \mathrm{S}_{24}$ & $9.67 \mathrm{def}$ & $14.67 \mathrm{def}$ & $91.33 \mathrm{a}-\mathrm{g}$ & $73.67 \mathrm{a}-\mathrm{g}$ & $76.67 \mathrm{c}-\mathrm{i}$ & $81.00 \mathrm{~b}-\mathrm{f}$ \\
\hline $\mathrm{V}_{2} \times \mathrm{T}_{2} \times \mathrm{S}_{24}$ & $10.33 \mathrm{c}-\mathrm{f}$ & $16.00 \mathrm{~b}-\mathrm{f}$ & $104.33 \mathrm{a}-\mathrm{f}$ & 75.67 a-d & 78.67 a-e & $82.00 \mathrm{a}-\mathrm{d}$ \\
\hline
\end{tabular}




\begin{tabular}{|l|c|c|c|c|c|c|}
\hline Treatment & $\begin{array}{c}\text { Days to 50\% } \\
\text { emergence }\end{array}$ & $\begin{array}{c}\text { Days to 90\% } \\
\text { emergence }\end{array}$ & $\begin{array}{c}\text { Plant population@ } \\
\mathbf{4 m}^{2}\end{array}$ & $\begin{array}{c}\text { Days to 1 } \\
\text { flowering }\end{array}$ & $\begin{array}{c}\text { Days to 50\% } \\
\text { flowering }\end{array}$ & $\begin{array}{c}\text { Days to 90\% } \\
\text { flowering }\end{array}$ \\
\hline $\mathrm{V}_{2} \times \mathrm{T}_{3} \times \mathrm{S}_{24}$ & $12.33 \mathrm{a}-\mathrm{d}$ & $16.00 \mathrm{~b}-\mathrm{f}$ & $93.33 \mathrm{a}-\mathrm{f}$ & $75.33 \mathrm{a}-\mathrm{d}$ & $79.00 \mathrm{a}-\mathrm{e}$ & $82.00 \mathrm{a}-\mathrm{d}$ \\
\hline $\mathrm{V}_{3} \times \mathrm{T}_{1} \times \mathrm{S}_{24}$ & $10.67 \mathrm{~b}-\mathrm{f}$ & $15.00 \mathrm{c}-\mathrm{f}$ & $94.67 \mathrm{a}-\mathrm{f}$ & $70.67 \mathrm{~g}-\mathrm{k}$ & $75.67 \mathrm{e}-\mathrm{i}$ & $78.00 \mathrm{ef}$ \\
\hline $\mathrm{V}_{3} \times \mathrm{T}_{2} \times \mathrm{S}_{24}$ & $11.00 \mathrm{a}-\mathrm{f}$ & $18.33 \mathrm{a}-\mathrm{d}$ & $69.00 \mathrm{fg}$ & $68.33 \mathrm{k}$ & $73.67 \mathrm{hi}$ & $77.67 \mathrm{f}$ \\
\hline $\mathrm{V}_{3} \times \mathrm{T}_{3} \times \mathrm{S}_{24}$ & $11.33 \mathrm{a}-\mathrm{f}$ & $18.67 \mathrm{abc}$ & $105.00 \mathrm{a}-\mathrm{f}$ & $70.00 \mathrm{~h}-\mathrm{k}$ & $74.33 \mathrm{~g}-\mathrm{i}$ & $78.33 \mathrm{ef}$ \\
\hline $\mathrm{V}_{4} \times \mathrm{T}_{1} \times \mathrm{S}_{24}$ & $9.00 \mathrm{ef}$ & $14.67 \mathrm{def}$ & $120.00 \mathrm{ab}$ & $77.00 \mathrm{a}$ & $80.00 \mathrm{abc}$ & $85.33 \mathrm{a}$ \\
\hline $\mathrm{V}_{4} \times \mathrm{T}_{2} \times \mathrm{S}_{24}$ & $14.00 \mathrm{a}$ & $20.67 \mathrm{a}$ & $85.00 \mathrm{~b}-\mathrm{g}$ & $76.67 \mathrm{ab}$ & $81.00 \mathrm{a}$ & $85.33 \mathrm{a}$ \\
\hline $\mathrm{V}_{4} \times \mathrm{T}_{3} \times \mathrm{S}_{24}$ & $13.33 \mathrm{abc}$ & $17.67 \mathrm{a}-\mathrm{e}$ & $55.67 \mathrm{~g}$ & $74.33 \mathrm{a}-\mathrm{f}$ & $79.33 \mathrm{a}-\mathrm{d}$ & $83.00 \mathrm{abc}$ \\
\hline $\mathrm{LSD}$ & 3.29 & 3.83 & 36.10 & 3.58 & 3.53 & 3.55 \\
\hline $\mathrm{LoS}$ & $*$ & $*$ & $*$ & $*$ & $*$ & $*$ \\
\hline $\mathrm{SEm}$ & 1.64 & 1.90 & 17.94 & 1.78 & 1.75 & 1.76 \\
\hline $\mathrm{CV}$ & $18.69 \%$ & $14.37 \%$ & $23.14 \%$ & $2.98 \%$ & $2.79 \%$ & $2.67 \%$ \\
\hline
\end{tabular}

Table 2: Interact effect of variety, fertilizer dose and soaking method on phenology and ontogenetic features of aus rice.

Means bearing same letter(s) in a column do not differ significantly at 5\% level of probability by LSD. LoS: Level of significance, SEm: Standard Error Mean, CV: Coefficient of Variation.

Gathered effect of the treatments pointed that, late $1^{\text {st }}$ and $90 \%$ flowering was belated in $\mathrm{V}_{4} \times \mathrm{T}_{1} \times \mathrm{S}_{24}$ (77.00 days and 85.33 days). Hence, $\mathrm{V}_{4} \times \mathrm{T}_{2} \times \mathrm{S}_{24}$ also delayed $50 \%$ and $90 \%$ flowering $(81.00$ days and 85.33 days). Earliest $1^{\text {st }}$ flowering was noticed with $\mathrm{V}_{3} \times$ $\mathrm{T}_{2} \times \mathrm{S}_{24}$ (68.33 days), $50 \%$ flowering in $\mathrm{V}_{3} \times \mathrm{T}_{3} \times \mathrm{S}_{0}$ (73.33 days) and $90 \%$ flowering in both $\mathrm{V}_{3} \times \mathrm{T}_{2} \times \mathrm{S}_{0}$ (77.67 days) and $\mathrm{V}_{3} \times \mathrm{T}_{2}$ $\times \mathrm{S}_{24}$ (77.67 days) treatment combinations. Flowering is relied on temperature, day length, and genetic character of the cultivar. Here, a mixed result was seen. BRRI dhan85 flowered late BRRI dhan82 flowered early. Treatments with recommended fertilizer dose and soaking application was found to have satisfactory results. Ghosh., et al. [40] stated flowering time in rice varieties fluctuated because of planting time and varietal attributes. Which more or less supported the present outcomes.

\section{Plant height and tillering dynamics}

Plant height of the rice varieties and tiller number per hill was significantly influenced by variety and fertilizer doses (Figure 5). Treatment $V_{3} \times T_{1}$ produced statistically longest $(108.67 \mathrm{~cm})$ and $V_{2}$ $\times \mathrm{T}_{1}$ shortest $(86.39 \mathrm{~cm})$ plant height. Ample number of total tillers (15.06) were seen with $V_{4} \times T_{3}$; whereas treatments $V_{2} \times T_{1}$ and $V_{2} \times$ $\mathrm{T}_{2}$ showed meagre tillers $(10.94 \mathrm{~cm}$ and $10.39 \mathrm{~cm})$. Number of noneffective tillers per hill was highest (2.06) in $\mathrm{V}_{2} \times \mathrm{T}_{3}$; but statistical identical and lowest number of non-effective tillers were recorded with treatments $\mathrm{V}_{1} \times \mathrm{T}_{3}(0.83)$ and $\mathrm{V}_{3} \times \mathrm{T}_{2}(0.83)$.

Varieties with different soaking and fertilizer with various soaking method interaction was detected (Figure 6). Highest plant

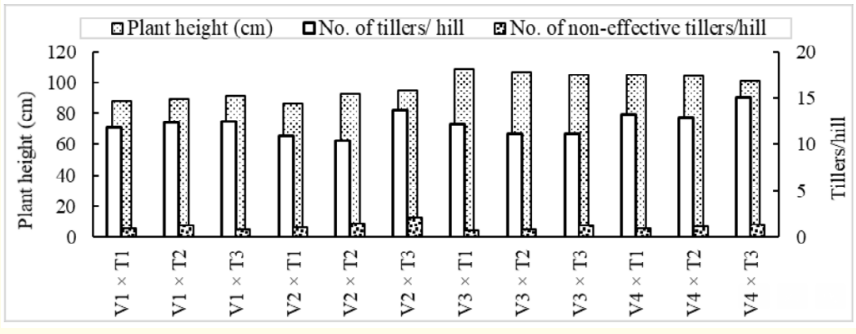

Figure 5: Plant height and tillers/hill as effected by variety and fertilizer.

height was noticed in treatments $\mathrm{V}_{3} \times \mathrm{S}_{0}(106.87 \mathrm{~cm})$ followed by $\mathrm{V}_{3}$ $\times \mathrm{S}_{24}(106.67 \mathrm{~cm}), \mathrm{V}_{4} \times \mathrm{S}_{24}(104.00 \mathrm{~cm})$ and $\mathrm{V}_{4} \times \mathrm{S}_{0}(103.11 \mathrm{~cm})$. Conversely, the lowest was witnessed with treatments $V_{1} \times S_{0}(88.33$ $\mathrm{cm})$ followed by $\mathrm{V}_{2} \times \mathrm{S}_{0}(90.11 \mathrm{~cm}), \mathrm{V}_{1} \times \mathrm{S}_{24}(90.59 \mathrm{~cm})$ and $\mathrm{V}_{2} \times \mathrm{S}_{24}$ $(92.82 \mathrm{~cm})$. Fertilizer and soaking methods had no significant interactions on plant height; i.e. they all were statistically same.

Maximum total number of tillers per hill (14.48) was obtained from treatment $\mathrm{V}_{4} \times \mathrm{S}_{24}$; while minimum from treatments $\mathrm{V}_{2} \times \mathrm{S}_{0}$ (10.26); followed by $V_{3} \times S_{24}(10.78)$ and $V_{1} \times S_{0}$ (11.03). Fertilizer dose and soaking application collaboration allowed treatment $\mathrm{T}_{3} \times$ $\mathrm{S}_{24}$ to produce profuse number of tillers (13.89) per hill and treatment $\mathrm{T}_{2} \times \mathrm{S}_{0}$ to generate sparse tillers (10.62). Contrary, number of non-effective tillers per hill was most (1.47) in treatment $T_{3} \times S_{24}$ 


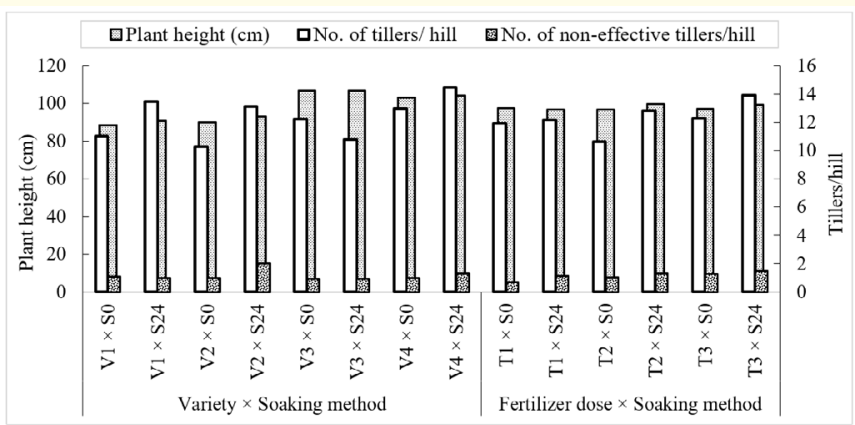

Figure 6: Influence of variety, fertilizer dose and soaking methods on height and tillering.

and least (0.67) in treatment $\mathrm{T}_{1} \times \mathrm{S}_{0}$ under this collaboration. With varietal and soaking impact, $\mathrm{V}_{2} \times \mathrm{S}_{24}$ treatment gave furthest number (2.00) of non-effective tillers per hill and the lowest (0.92) was attained by $\mathrm{T}_{3} \times \mathrm{S}_{24}$.

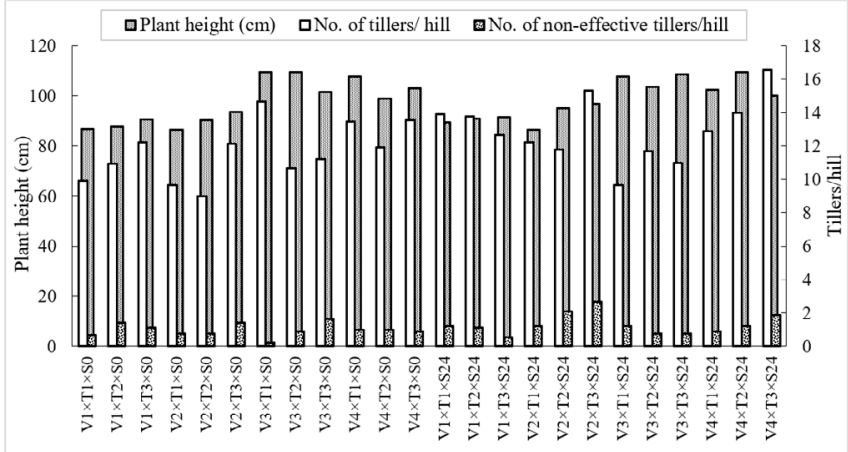

Figure 7: Collective effect of variety, fertilizer and seed soaking on height and tillering.

Aggregative effect of variety, fertilizer and soaking had much distinctions on the height and tillering attributes of aus rice varieties (Figure 7). Maximum $109.56 \mathrm{~cm}$ height was attained from three treatments $\left(\mathrm{V}_{3} \times \mathrm{T}_{1} \times \mathrm{S}_{0,} \mathrm{~V}_{3} \times \mathrm{T}_{2} \times \mathrm{S}_{0}\right.$ and $\left.\mathrm{V}_{4} \times \mathrm{T}_{2} \times \mathrm{S}_{24}\right)$. On contrary, statistically equal minimum height was also gained from three treatments i.e. $\mathrm{V}_{2} \times \mathrm{T}_{1} \times \mathrm{S}_{0}(86.33 \mathrm{~cm}), \mathrm{V}_{1} \times \mathrm{T}_{1} \times \mathrm{S}_{0}(86.78$ $\mathrm{cm})$ and $\mathrm{V}_{2} \times \mathrm{T}_{1} \times \mathrm{S}_{0}(86.45 \mathrm{~cm})$; all of them carried the same letter. Maximal number of tillers per hill was recorded in $V_{4} \times T_{3} \times S_{24}$
(16.56) and fewest were found with treatment $V_{2} \times T_{2} \times S_{0}(9.00)$. Treatment $\mathrm{V}_{2} \times \mathrm{T}_{3} \times \mathrm{S}_{24}$ had the greatest number of non-effective tillers per hill (2.67) and $\mathrm{V}_{3} \times \mathrm{T}_{1} \times \mathrm{S}_{0}(0.22)$ treatment had the least. Generally, dwarf plant architecture is desired during the aus season as this time faces natural calamities such as heavy wind, rainfall, storm, hail storm etc. Mean plant height of BRRI dhan82 and BRRI dhan85 is $110 \mathrm{~cm}[41,42]$. Average height of Binadhan-19 and Binadhan-21 is around $100 \mathrm{~cm}[43,44]$. Shorter height was might be due to direct seeding cultivation. $80 \%$ and $100 \%$ recommended fertilizer dose with no priming resulted in longer plant height; but minimum height was might be no priming and low fertilizer application in the plots. Higher fertilizer dose and priming ensured better tillering capability and vice versa. Mohanta., et al. [15] alluded that, plant height, number of tillers per $\mathrm{m}^{2}$ and effective tillers $\mathrm{m}^{-2}$ is related to crop establishment, Nitrogen fertilizer management practice in field.

\section{Panicle attributes}

Effect of variety-fertilizer, variety-soaking and variety-fertilizersoaking was significantly influenced on panicle number, panicle length and panicle weight. But these parameters were unaffected by fertilizer-soaking interaction (Table 3, Table 4).

Number of panicles per hill was utmost in treatment $\mathrm{V}_{4} \times \mathrm{T}_{3}$ (13.72) and lowest in $V_{2} \times T_{2}$ (8.95). In next effect, treatment $V_{4}$ $\times \mathrm{S}_{24}(13.19)$ attained highest amount of panicles per hill and the least was gained from $\mathrm{V}_{2} \times \mathrm{S}_{0}$ (9.22). Interaction effect of all the treatments demonstrated that, treatment $\mathrm{V}_{4} \times \mathrm{T}_{3} \times \mathrm{S}_{24}$ and $\mathrm{V}_{3} \times \mathrm{T}_{1}$ $\times \mathrm{S}_{0}$ gave more number of panicles (14.48 and 14.43) than others. Seven treatment combinations showed lesser and statistical identical number of panicles; among them $\mathrm{V}_{2} \times \mathrm{T}_{2} \times \mathrm{S}_{0}$ was the least (8.22).

Extended panicle length $(26.90 \mathrm{~cm})$ was noticed in treatment $\mathrm{V}_{3} \times \mathrm{T}_{1}$. While $\mathrm{V}_{1} \times \mathrm{T}_{1}$ and $\mathrm{V}_{1} \times \mathrm{T}_{2}$ treatments had narrowed length $(22.06 \mathrm{~cm}$ and $21.95 \mathrm{~cm})$ panicle. Lengthy panicle $(25.85 \mathrm{~cm})$ was seen with treatment $\mathrm{V}_{3} \times \mathrm{S}_{0}$. Whereas $\mathrm{V}_{1} \times \mathrm{S}_{24}$ and $\mathrm{V}_{1} \times \mathrm{S}_{0}$ treatments demonstrated reduced sized $(22.10 \mathrm{~cm}$ and $22.33 \mathrm{~cm})$ panicle. Collaborative effect of all the treatments indicated $\mathrm{V}_{3} \times \mathrm{T}_{1} \times \mathrm{S}_{24}$ had more elongated $(27.14 \mathrm{~cm})$ panicle than others; shortened alike length of panicles were observed in $\mathrm{V}_{1} \times \mathrm{T}_{1} \times \mathrm{S}_{0}(21.74 \mathrm{~cm})$ and $\mathrm{V}_{1}$ $\times \mathrm{T}_{2} \times \mathrm{S}_{24}(21.78 \mathrm{~cm})$. 
Panicle weight was maximum in treatment $\mathrm{V}_{3} \times \mathrm{T}_{1}(10.40 \mathrm{~g})$ and $\mathrm{V}_{3} \times \mathrm{S}_{24}(9.39 \mathrm{~g})$. While the minimum was noted with $\mathrm{V}_{1} \times \mathrm{T}_{2}(6.23 \mathrm{~g})$ and $\mathrm{V}_{1} \times \mathrm{S}_{24}(6.44 \mathrm{~g})$ treatment.

Collective effect of all the factors revealed that, $V_{3} \times T_{1} \times S_{24}$ treatment gave heaviest (10.49 g) panicle weight and the lightest was seen with treatment $\mathrm{V}_{1} \times \mathrm{T}_{2} \times \mathrm{S}_{24}(5.77 \mathrm{~g})$. BRRI dhan82 and BRRI dhan 85 had more number of panicles under low and high fertilizer dose. Presumably BRRI dhan85 was more responsive to fertilizer and priming than other varieties. However, treatments with least panicle numbers produced more panicle length and weight in most interaction effects. Panicle length being a genetic trait might have altered due to crop establish method and nutrient utilization from soil. Panicle weight was increased with panicle length. Jnanesha and Kumar [45] ascribed panicle length relied on crop establishment. Xu., et al. [46] reported that panicle number $\mathrm{m}^{-2}$ and seed weight is positively related on soil fertility.
Root length

Longest root length was noted in treatments $V_{2} \times T_{2}\left(12.11 \mathrm{~cm}^{2}\right)$ and $V_{2} \times S_{24}\left(12.76 \mathrm{~cm}^{2}\right)$. Whereas, shortest length was seen with treatments $\mathrm{V}_{4} \times \mathrm{T}_{3}\left(10.03 \mathrm{~cm}^{2}\right)$ and $\mathrm{V}_{4} \times \mathrm{T}_{3}\left(10.39 \mathrm{~cm}^{2}\right)$. Fertilizer and soaking method gave statistically non-significant result and thus remained unaffected by the factors. Interaction effect of the factors elucidated that, treatment $\mathrm{V}_{2} \times \mathrm{T}_{2} \times \mathrm{S}_{24}$ had utmost root length $\left(13.50 \mathrm{~cm}^{2}\right)$ and treatments $\mathrm{V}_{4} \times \mathrm{T}_{3} \times \mathrm{S}_{0}\left(9.56 \mathrm{~cm}^{2}\right)$ and $\mathrm{V}_{4} \times$ $\mathrm{T}_{1} \times \mathrm{S}_{24}\left(9.59 \mathrm{~cm}^{2}\right)$ delivered lowest root area (Table 3 , Table 4$)$. Binadhan-21 was derived from NERICA-4; which was more tolerant to drought and thereby more root area was noticed. BRRI dhan85 might have the capability to utilize extra fertilizer but Binadhan-21 performed better in recommended dose. Mahajan., et al. [47] inferred that, root length in DSR was connected to crop establishment and seed priming methods.

\begin{tabular}{|c|c|c|c|c|c|c|c|}
\hline Treat. & Panicles/ hill & $\begin{array}{l}\text { Panicle } \\
\text { length } \\
\text { (cm) }\end{array}$ & $\begin{array}{c}\text { Panicle } \\
\text { weight (g) }\end{array}$ & $\begin{array}{l}\text { Root length } \\
\qquad\left(\mathrm{cm}^{2}\right)\end{array}$ & Filled grains/ panicle & $\begin{array}{c}\text { Unfilled } \\
\text { grains/panicle } \\
\text { (\%) }\end{array}$ & $\begin{array}{l}1000 \text { grain } \\
\text { weight (g) }\end{array}$ \\
\hline \multicolumn{7}{|c|}{ Variety $\times$ Fertilizer dose } & \\
\hline $\mathrm{V}_{1} \times \mathrm{T}_{1}$ & $10.83 \mathrm{abc}$ & $22.06 \mathrm{e}$ & $7.03 \mathrm{bcd}$ & $11.08 \mathrm{ab}$ & $167.43 \mathrm{bcd}$ & $33.73 \mathrm{~cd}$ & $22.56 \mathrm{ab}$ \\
\hline $\mathrm{V}_{1} \times \mathrm{T}_{2}$ & $12.61 \mathrm{ab}$ & $21.95 \mathrm{e}$ & $6.23 \mathrm{~d}$ & $12.06 \mathrm{ab}$ & 129.38 ef & $36.37 \mathrm{a}-\mathrm{d}$ & $23.40 \mathrm{a}$ \\
\hline $\mathrm{V}_{1} \times \mathrm{T}_{3}$ & $11.61 \mathrm{abc}$ & $22.63 \mathrm{de}$ & $7.49 \mathrm{bcd}$ & $10.54 \mathrm{ab}$ & $144.94 \mathrm{c}-\mathrm{f}$ & 37.69 a-d & $21.94 \mathrm{a}-\mathrm{d}$ \\
\hline $\mathrm{V}_{2} \times \mathrm{T}_{1}$ & $9.89 \mathrm{bc}$ & 23.27 cde & $8.81 \mathrm{ab}$ & $12.03 \mathrm{ab}$ & $174.85 \mathrm{bcd}$ & $32.93 \mathrm{~cd}$ & $21.51 \mathrm{bcd}$ \\
\hline $\mathrm{V}_{2} \times \mathrm{T}_{2}$ & $8.95 \mathrm{c}$ & $23.99 \mathrm{bcd}$ & $8.51 \mathrm{abc}$ & $12.11 \mathrm{a}$ & $178.65 \mathrm{bc}$ & $39.38 \mathrm{abc}$ & $20.44 \mathrm{~d}$ \\
\hline $\mathrm{V}_{2} \times \mathrm{T}_{3}$ & $11.67 \mathrm{abc}$ & $23.80 \mathrm{~cd}$ & $8.09 \mathrm{bcd}$ & $12.05 \mathrm{ab}$ & $141.44 \mathrm{def}$ & $44.93 \mathrm{a}$ & $21.26 \mathrm{bcd}$ \\
\hline $\mathrm{V}_{3} \times \mathrm{T}_{1}$ & $11.44 \mathrm{abc}$ & $26.90 \mathrm{a}$ & $10.40 \mathrm{a}$ & $10.56 \mathrm{ab}$ & $197.49 \mathrm{ab}$ & $35.28 \mathrm{bcd}$ & $20.46 \mathrm{~d}$ \\
\hline $\mathrm{V}_{3} \times \mathrm{T}_{2}$ & $9.78 \mathrm{bc}$ & $25.45 \mathrm{ab}$ & $8.70 \mathrm{ab}$ & $10.23 \mathrm{ab}$ & 133.37 ef & $39.98 \mathrm{abc}$ & $22.26 \mathrm{abc}$ \\
\hline $\mathrm{V}_{3} \times \mathrm{T}_{3}$ & $9.89 \mathrm{bc}$ & $25.38 \mathrm{~b}$ & $7.56 \mathrm{bcd}$ & $11.16 \mathrm{ab}$ & 153.32 cde & $43.09 \mathrm{ab}$ & $21.98 \mathrm{a}-\mathrm{d}$ \\
\hline $\mathrm{V}_{4} \times \mathrm{T}_{1}$ & $12.22 \mathrm{abc}$ & $23.65 \mathrm{~cd}$ & $6.60 \mathrm{~cd}$ & $10.65 \mathrm{ab}$ & 223.18 a & $29.57 \mathrm{~d}$ & $20.56 \mathrm{~cd}$ \\
\hline$V_{4} \times T_{2}$ & $11.83 \mathrm{abc}$ & $23.74 \mathrm{~cd}$ & $7.08 \mathrm{bcd}$ & $10.64 \mathrm{ab}$ & $119.17 \mathrm{f}$ & $40.86 \mathrm{abc}$ & $20.86 \mathrm{bcd}$ \\
\hline $\mathrm{V}_{4} \times \mathrm{T}_{3}$ & $13.72 \mathrm{a}$ & $24.45 \mathrm{bc}$ & $7.92 \mathrm{bcd}$ & $10.03 \mathrm{~b}$ & $146.21 \mathrm{c}-\mathrm{f}$ & $40.47 \mathrm{abc}$ & $21.05 \mathrm{bcd}$ \\
\hline $\mathrm{LSD}_{0.05}$ & 3.36 & 1.46 & 1.97 & 2.07 & 33.99 & 8.58 & 1.79 \\
\hline LoS & $*$ & $*$ & $*$ & $*$ & $*$ & $*$ & $*$ \\
\hline SEm \pm & 1.67 & 0.72 & 0.98 & 1.03 & 16.89 & 4.26 & 0.89 \\
\hline
\end{tabular}




\begin{tabular}{|c|c|c|c|c|c|c|c|}
\hline Treat. & Panicles/ hill & $\begin{array}{c}\text { Panicle } \\
\text { length } \\
\text { (cm) }\end{array}$ & $\begin{array}{c}\text { Panicle } \\
\text { weight (g) }\end{array}$ & $\begin{array}{c}\text { Root length } \\
\left(\mathrm{cm}^{2}\right)\end{array}$ & Filled grains/ panicle & $\begin{array}{c}\text { Unfilled } \\
\text { grains/panicle } \\
\text { (\%) }\end{array}$ & $\begin{array}{c}1000 \text { grain } \\
\text { weight }(\mathrm{g})\end{array}$ \\
\hline \multicolumn{8}{|c|}{ Variety $\times$ Soaking method } \\
\hline $\mathrm{V}_{1} \times \mathrm{S}_{0}$ & $10.89 \mathrm{abc}$ & $22.33 \mathrm{c}$ & 7.38 bcd & $10.82 \mathrm{~b}$ & $155.59 \mathrm{bc}$ & $36.14 \mathrm{ab}$ & $22.43 \mathrm{ab}$ \\
\hline $\mathrm{V}_{1} \times \mathrm{S}_{24}$ & $12.48 \mathrm{ab}$ & $22.10 \mathrm{c}$ & $6.44 \mathrm{~d}$ & $11.63 \mathrm{ab}$ & $138.90 \mathrm{~cd}$ & $35.72 \mathrm{ab}$ & 22.83 a \\
\hline $\mathrm{V}_{2} \times \mathrm{S}_{0}$ & $9.22 \mathrm{c}$ & $23.16 \mathrm{bc}$ & $8.16 \mathrm{abc}$ & $11.37 \mathrm{ab}$ & $144.97 \mathrm{~cd}$ & $41.11 \mathrm{a}$ & $20.81 \mathrm{c}$ \\
\hline $\mathrm{V}_{2} \times \mathrm{S}_{24}$ & $11.11 \mathrm{abc}$ & $24.22 \mathrm{~b}$ & $8.77 \mathrm{ab}$ & $12.76 \mathrm{a}$ & $184.99 \mathrm{a}$ & $37.05 \mathrm{ab}$ & $21.32 \mathrm{bc}$ \\
\hline $\mathrm{V}_{3} \times \mathrm{S}_{0}$ & $10.89 \mathrm{abc}$ & $25.85 \mathrm{a}$ & $8.38 \mathrm{abc}$ & $10.39 \mathrm{~b}$ & $178.20 \mathrm{ab}$ & $39.11 \mathrm{ab}$ & $21.66 \mathrm{abc}$ \\
\hline $\mathrm{V}_{3} \times \mathrm{S}_{24}$ & $9.85 \mathrm{bc}$ & $25.96 \mathrm{a}$ & $9.39 \mathrm{a}$ & $10.90 \mathrm{~b}$ & $126.89 \mathrm{~d}$ & $42.52 \mathrm{a}$ & $21.47 \mathrm{abc}$ \\
\hline $\mathrm{V}_{4} \times \mathrm{S}_{0}$ & $12.00 \mathrm{ab}$ & $23.97 \mathrm{~b}$ & $7.30 \mathrm{bcd}$ & $10.46 \mathrm{~b}$ & $204.46 \mathrm{a}$ & $32.84 \mathrm{~b}$ & 20.81 c \\
\hline $\mathrm{V}_{4} \times \mathrm{S}_{24}$ & $13.19 \mathrm{a}$ & $23.92 \mathrm{~b}$ & $7.10 \mathrm{~cd}$ & $10.42 \mathrm{~b}$ & $138.94 \mathrm{~cd}$ & $38.35 \mathrm{ab}$ & $20.83 \mathrm{c}$ \\
\hline $\operatorname{LSD}_{0.05}$ & 2.74 & 1.19 & 1.61 & 1.69 & 27.76 & 7.00 & 1.46 \\
\hline LoS & $*$ & $*$ & $*$ & $*$ & $*$ & $*$ & $*$ \\
\hline SEm \pm & 1.36 & 0.59 & 0.80 & 0.84 & 13.79 & 3.48 & 0.72 \\
\hline \multicolumn{8}{|c|}{ Fertilizer dose $\times$ Soaking method } \\
\hline $\mathrm{T}_{1} \times \mathrm{S}_{0}$ & 11.17 & 23.77 & 8.24 & 10.99 & $170.77 \mathrm{a}$ & $33.40 \mathrm{~b}$ & 21.06 \\
\hline $\mathrm{T}_{1} \times \mathrm{S}_{24}$ & 11.03 & 24.17 & 8.16 & 11.16 & $165.80 \mathrm{a}$ & $37.55 \mathrm{~b}$ & 21.48 \\
\hline $\mathrm{T}_{2} \times \mathrm{S}_{0}$ & 10.08 & 23.66 & 7.65 & 10.53 & $151.33 \mathrm{ab}$ & $37.23 \mathrm{~b}$ & 21.82 \\
\hline $\mathrm{T}_{2} \times \mathrm{S}_{24}$ & 11.50 & 23.91 & 7.60 & 11.99 & $173.86 \mathrm{a}$ & $35.85 \mathrm{~b}$ & 21.66 \\
\hline $\mathrm{T}_{3} \times \mathrm{S}_{0}$ & 11.00 & 24.06 & 7.52 & 10.76 & $137.12 \mathrm{~b}$ & $44.37 \mathrm{a}$ & 21.41 \\
\hline $\mathrm{T}_{3} \times \mathrm{S}_{24}$ & 12.44 & 24.08 & 8.01 & 11.14 & $155.84 \mathrm{ab}$ & $38.72 \mathrm{ab}$ & 21.71 \\
\hline $\mathrm{LSD}_{0.05}$ & 2.38 & 1.03 & 1.39 & 1.47 & 24.04 & 6.07 & 1.26 \\
\hline LoS & NS & NS & NS & NS & $*$ & $*$ & NS \\
\hline SEm \pm & 1.18 & 0.51 & 0.69 & 0.73 & 11.94 & 3.01 & 0.63 \\
\hline $\mathrm{CV}$ & $25.81 \%$ & $5.24 \%$ & $21.53 \%$ & $16.08 \%$ & $18.38 \%$ & $19.50 \%$ & $7.14 \%$ \\
\hline
\end{tabular}

Table 3: Yield components of aus rice influenced by variety, fertilizer dose and soaking.

Means bearing same letter(s) in a column do not differ significantly at 5\% level of probability by LSD. LoS: Level of Significance, SEm: Standard Error Mean, NS: Non-significant, CV: Coefficient of Variation.

Grains per panicle and TGW

Consequence of variety-fertilizer, variety-soaking, fertilizersoaking (except TGW) and variety-fertilizer-soaking (collective interaction) significantly influenced number of grains per panicle and 1000 grain weight (Table 3, Table 4).

\begin{tabular}{|l|c|c|c|c|c|c|c|}
\hline Treatment & Panicles/hill & $\begin{array}{c}\text { Panicle } \\
\text { length } \\
\mathbf{( c m )}\end{array}$ & $\begin{array}{c}\text { Panicle } \\
\text { weight }(\mathbf{g})\end{array}$ & $\begin{array}{c}\text { Root length } \\
\mathbf{( \mathbf { c m } ^ { 2 } )}\end{array}$ & Filled grains/ panicle & $\begin{array}{c}\text { Unfilled grains/ } \\
\text { panicle (\%) }\end{array}$ & $\begin{array}{c}\text { 1000 grain } \\
\text { weight (g) }\end{array}$ \\
\hline $\mathrm{V}_{1} \times \mathrm{T}_{1} \times \mathrm{S}_{0}$ & $9.00 \mathrm{~b}$ & $21.74 \mathrm{~g}$ & $7.15 \mathrm{cde}$ & $10.33 \mathrm{bc}$ & $190.41 \mathrm{a}-\mathrm{d}$ & $33.09 \mathrm{c}-\mathrm{f}$ & $22.32 \mathrm{a}-\mathrm{d}$ \\
\hline $\mathrm{V}_{1} \times \mathrm{T}_{2} \times \mathrm{S}_{0}$ & $12.56 \mathrm{ab}$ & $22.13 \mathrm{fg}$ & $6.70 \mathrm{cde}$ & $11.44 \mathrm{abc}$ & $127.04 \mathrm{fgh}$ & $37.39 \mathrm{a}-\mathrm{f}$ & $23.07 \mathrm{ab}$ \\
\hline $\mathrm{V}_{1} \times \mathrm{T}_{3} \times \mathrm{S}_{0}$ & $11.11 \mathrm{ab}$ & $23.12 \mathrm{~d}-\mathrm{g}$ & $8.30 \mathrm{a}-\mathrm{e}$ & $10.69 \mathrm{abc}$ & $149.33 \mathrm{~d}-\mathrm{g}$ & $37.94 \mathrm{a}-\mathrm{f}$ & $21.91 \mathrm{a}-\mathrm{e}$ \\
\hline $\mathrm{V}_{2} \times \mathrm{T}_{1} \times \mathrm{S}_{0}$ & $8.78 \mathrm{~b}$ & $22.83 \mathrm{efg}$ & $8.39 \mathrm{a}-\mathrm{e}$ & $11.61 \mathrm{abc}$ & $151.49 \mathrm{c}-\mathrm{g}$ & $36.76 \mathrm{a}-\mathrm{f}$ & $21.08 \mathrm{~b}-\mathrm{e}$ \\
\hline $\mathrm{V}_{2} \times \mathrm{T}_{2} \times \mathrm{S}_{0}$ & $8.22 \mathrm{~b}$ & $23.09 \mathrm{~d}-\mathrm{g}$ & $8.82 \mathrm{abcd}$ & $10.72 \mathrm{abc}$ & $167.64 \mathrm{~b}-\mathrm{f}$ & $38.39 \mathrm{a}-\mathrm{f}$ & $21.29 \mathrm{a}-\mathrm{e}$ \\
\hline
\end{tabular}




\begin{tabular}{|c|c|c|c|c|c|c|c|}
\hline Treatment & Panicles/hill & $\begin{array}{c}\text { Panicle } \\
\text { length } \\
\text { (cm) }\end{array}$ & $\begin{array}{c}\text { Panicle } \\
\text { weight (g) }\end{array}$ & $\begin{array}{l}\text { Root length } \\
\left(\mathrm{cm}^{2}\right)\end{array}$ & Filled grains/ panicle & $\begin{array}{c}\text { Unfilled grains/ } \\
\text { panicle (\%) }\end{array}$ & $\begin{array}{l}1000 \text { grain } \\
\text { weight }(\mathrm{g})\end{array}$ \\
\hline $\mathrm{V}_{2} \times \mathrm{T}_{3} \times \mathrm{S}_{0}$ & $10.67 \mathrm{ab}$ & $23.55 \mathrm{~d}-\mathrm{g}$ & $7.28 \mathrm{cde}$ & $11.78 \mathrm{abc}$ & $115.79 \mathrm{gh}$ & 48.17 a & $20.08 \mathrm{de}$ \\
\hline $\mathrm{V}_{3} \times \mathrm{T}_{1} \times \mathrm{S}_{0}$ & $14.43 \mathrm{a}$ & $26.65 \mathrm{ab}$ & $10.30 \mathrm{ab}$ & $10.33 \mathrm{bc}$ & 186.45 b-e & 39.18 a-f & 20.34 cde \\
\hline $\mathrm{V}_{3} \times \mathrm{T}_{2} \times \mathrm{S}_{0}$ & $8.66 \mathrm{~b}$ & $26.11 \mathrm{abc}$ & 8.04 a-e & $9.85 \mathrm{bc}$ & $209.40 \mathrm{ab}$ & $31.73 \mathrm{def}$ & $22.12 \mathrm{a}-\mathrm{d}$ \\
\hline $\mathrm{V}_{3} \times \mathrm{T}_{3} \times \mathrm{S}_{0}$ & $9.56 \mathrm{~b}$ & 24.80 b-e & $6.80 \mathrm{cde}$ & $10.99 \mathrm{abc}$ & $138.75 \mathrm{e}-\mathrm{h}$ & $46.43 \mathrm{ab}$ & $22.51 \mathrm{a}-\mathrm{d}$ \\
\hline $\mathrm{V}_{4} \times \mathrm{T}_{1} \times \mathrm{S}_{0}$ & $12.44 \mathrm{ab}$ & $23.85 \mathrm{def}$ & 7.14 cde & $11.72 \mathrm{abc}$ & $236.96 \mathrm{a}$ & $27.40 \mathrm{f}$ & 20.52 cde \\
\hline $\mathrm{V}_{4} \times \mathrm{T}_{2} \times \mathrm{S}_{0}$ & $10.89 \mathrm{ab}$ & $23.31 \mathrm{~d}-\mathrm{g}$ & $7.08 \mathrm{cde}$ & $10.10 \mathrm{bc}$ & $101.24 \mathrm{~h}$ & $41.42 \mathrm{a}-\mathrm{d}$ & 20.79 b-e \\
\hline $\mathrm{V}_{4} \times \mathrm{T}_{3} \times \mathrm{S}_{0}$ & $12.67 \mathrm{ab}$ & 24.74 b-e & $7.69 \mathrm{~b}-\mathrm{e}$ & $9.56 \mathrm{c}$ & $144.62 \mathrm{~d}-\mathrm{h}$ & $44.96 \mathrm{abc}$ & $21.12 \mathrm{~b}-\mathrm{e}$ \\
\hline $\mathrm{V}_{1} \times \mathrm{T}_{1} \times \mathrm{S}_{24}$ & $12.66 \mathrm{ab}$ & $22.38 \mathrm{fg}$ & $6.90 \mathrm{cde}$ & $11.83 \mathrm{abc}$ & $144.45 \mathrm{~d}-\mathrm{h}$ & $34.36 \mathrm{~b}-\mathrm{f}$ & $22.81 \mathrm{abc}$ \\
\hline $\mathrm{V}_{1} \times \mathrm{T}_{2} \times \mathrm{S}_{24}$ & $12.67 \mathrm{ab}$ & $21.78 \mathrm{~g}$ & $5.77 \mathrm{e}$ & $12.67 \mathrm{ab}$ & $131.71 \mathrm{fgh}$ & $35.34 \mathrm{~b}-\mathrm{f}$ & $23.73 \mathrm{a}$ \\
\hline $\mathrm{V}_{1} \times \mathrm{T}_{3} \times \mathrm{S}_{24}$ & $12.11 \mathrm{ab}$ & $22.13 \mathrm{fg}$ & 6.67 cde & $10.39 \mathrm{bc}$ & $140.56 \mathrm{e}-\mathrm{h}$ & 37.44 a-f & 21.97 a-e \\
\hline $\mathrm{V}_{2} \times \mathrm{T}_{1} \times \mathrm{S}_{24}$ & $11.00 \mathrm{ab}$ & $23.70 \mathrm{~d}-\mathrm{g}$ & $9.22 \mathrm{abc}$ & $12.44 \mathrm{abc}$ & $198.20 \mathrm{abc}$ & 29.10 ef & 21.94 a-e \\
\hline $\mathrm{V}_{2} \times \mathrm{T}_{2} \times \mathrm{S}_{24}$ & $9.67 \mathrm{~b}$ & $24.91 \mathrm{bcd}$ & 8.20 a-e & $13.50 \mathrm{a}$ & 189.66 a-d & 40.36 a-e & 19.59 e \\
\hline $\mathrm{V}_{2} \times \mathrm{T}_{3} \times \mathrm{S}_{24}$ & $12.67 \mathrm{ab}$ & $24.05 \mathrm{c}-\mathrm{f}$ & $8.89 a b c$ & $12.33 \mathrm{abc}$ & $167.09 \mathrm{~b}-\mathrm{f}$ & $41.68 \mathrm{a}-\mathrm{d}$ & $22.45 \mathrm{a}-\mathrm{d}$ \\
\hline $\mathrm{V}_{3} \times \mathrm{T}_{1} \times \mathrm{S}_{24}$ & $8.44 \mathrm{~b}$ & $27.14 \mathrm{a}$ & 10.49 a & $10.78 \mathrm{abc}$ & $208.53 \mathrm{ab}$ & $31.37 \mathrm{def}$ & 20.58 b-e \\
\hline $\mathrm{V}_{3} \times \mathrm{T}_{2} \times \mathrm{S}_{24}$ & $10.89 \mathrm{ab}$ & 24.78 b-e & $9.36 \mathrm{abc}$ & $10.61 \mathrm{abc}$ & $134.83 \mathrm{fgh}$ & 41.19 a-e & $22.40 \mathrm{a}-\mathrm{d}$ \\
\hline $\mathrm{V}_{3} \times \mathrm{T}_{3} \times \mathrm{S}_{24}$ & $10.22 \mathrm{ab}$ & $25.95 \mathrm{abc}$ & 8.33 a-e & $11.33 \mathrm{abc}$ & 167.89 b-f & 39.76 a-e & 21.44 a-e \\
\hline $\mathrm{V}_{4} \times \mathrm{T}_{1} \times \mathrm{S}_{24}$ & $12.00 \mathrm{ab}$ & $23.45 \mathrm{~d}-\mathrm{g}$ & $6.06 \mathrm{de}$ & $9.59 \mathrm{c}$ & $131.91 \mathrm{fgh}$ & 38.78 a-f & 20.59 b-e \\
\hline $\mathrm{V}_{4} \times \mathrm{T}_{2} \times \mathrm{S}_{24}$ & $12.78 \mathrm{ab}$ & $24.16 \mathrm{c}-\mathrm{f}$ & $7.08 \mathrm{cde}$ & $11.18 \mathrm{abc}$ & $137.10 \mathrm{fgh}$ & 40.31 a-e & 20.92 b-e \\
\hline $\mathrm{V}_{4} \times \mathrm{T}_{3} \times \mathrm{S}_{24}$ & $14.78 \mathrm{a}$ & $24.16 \mathrm{c}-\mathrm{f}$ & 8.15 a-e & $10.50 \mathrm{bc}$ & $147.81 \mathrm{~d}-\mathrm{h}$ & 35.98 b-f & 20.98 b-e \\
\hline $\mathrm{LSD}_{0.05}$ & 4.75 & 2.06 & 2.78 & 2.93 & 48.08 & 12.13 & 2.53 \\
\hline LoS & $*$ & $*$ & $*$ & $*$ & ${ }^{*}$ & $*$ & $*$ \\
\hline SEm \pm & 2.36 & 1.02 & 1.38 & 1.46 & 23.89 & 6.03 & 1.25 \\
\hline $\mathrm{CV}$ & $25.81 \%$ & $5.24 \%$ & $21.53 \%$ & $16.08 \%$ & $18.38 \%$ & $19.50 \%$ & $7.14 \%$ \\
\hline
\end{tabular}

Table 4: Interaction effect of variety, fertilizer dose and soaking method on the yield attributes of aus rice.

Means bearing same letter(s) in a column do not differ significantly at 5\% level of probability by LSD. LoS: Level of Significance, SEm: Standard Error Mean, CV: Coefficient of Variation.

Number of filled grains per panicle were maximum in treatments $\mathrm{V}_{4} \times \mathrm{T}_{1}$ (223.18), $\mathrm{V}_{4} \times \mathrm{S}_{0}(204.46)$ and $\mathrm{T}_{1} \times \mathrm{S}_{0}$ (170.77). Contrary, minimum was noted with treatments $\mathrm{V}_{4} \times \mathrm{T}_{2}(119.17), \mathrm{V}_{3} \times \mathrm{S}_{24}$ (126.89) and $\mathrm{T}_{3} \times \mathrm{S}_{0}$ (137.12). Interaction of factors showed treatment $\mathrm{V}_{4} \times \mathrm{T}_{1} \times \mathrm{S}_{0}$ had most (236.96) filled grains and $\mathrm{V}_{4} \times \mathrm{T}_{2} \times \mathrm{S}_{0}$ (101.24) had least amount of filled grains per panicle.
Percentage of unfilled grains per panicle was abundant in treatments $\mathrm{V}_{2} \times \mathrm{T}_{3}(44.93 \%), \mathrm{V}_{3} \times \mathrm{S}_{24}(42.52 \%)$ and $\mathrm{T}_{3} \times \mathrm{S}_{0}(44.37 \%)$. While scarce grain sterility was observed in treatments $\mathrm{V}_{4} \times \mathrm{T}_{1}$ (29.57\%), $\mathrm{V}_{4} \times \mathrm{S}_{0}(32.84 \%)$ and $\mathrm{T}_{1} \times \mathrm{S}_{0}(33.40 \%)$. Combined factorial effect demonstrated that greatest sterile grains were produced by treatment $\mathrm{V}_{2} \times \mathrm{T}_{3} \times \mathrm{S}_{0}(48.17 \%)$ and the little sterility was spotted with treatment $\mathrm{V}_{4} \times \mathrm{T}_{1} \times \mathrm{S}_{0}(27.40 \%)$. 
1000 grain weight (TGW) was maximum in treatments $\mathrm{V}_{1} \times \mathrm{T}_{1}$ (23.40 g), $\mathrm{V}_{1} \times \mathrm{S}_{24}(22.83 \mathrm{~g})$. Whereas minimum TGW was noticed with treatments $\mathrm{V}_{2} \times \mathrm{T}_{2}(20.44 \mathrm{~g}), \mathrm{V}_{3} \times \mathrm{T}_{1}(20.46 \mathrm{~g}), \mathrm{V}_{2} \times \mathrm{S}_{0}(20.81$ g), $\mathrm{V}_{4} \times \mathrm{S}_{0}(20.81 \mathrm{~g})$ and $\mathrm{V}_{4} \times \mathrm{S}_{24}(20.83 \mathrm{~g})$. TGW remained uninfluenced by fertilizer-soaking interaction and gave non-significant result. Aggregative effect of variety-fertilizer-soaking revealed that, heaviest TGW was found with treatment $\mathrm{V}_{1} \times \mathrm{T}_{2} \times \mathrm{S}_{24}(23.73 \mathrm{~g})$ and lightest was seen by $\mathrm{V}_{2} \times \mathrm{T}_{2} \times \mathrm{S}_{24}(19.59 \mathrm{~g})$. Observations disclosed that filled grains were least with more fertilizer and more with less fertilizer. Therefore, high amount of grain sterility indicates that fertilizer management is crucial in DSR production. Normally rice gains $15-20 \%$ sterile grains [48]; but in DSR higher sterility was found. Overall, excess fertilizer adversely affected filled and caused more sterile grains. TGW is a varietal trait which reflects the bold or premium quality of the grain. TGW of Binadhan-19 was more and Binadhan-21 was less which represent slender type grain. However, in the experiment TGW might have influenced from nutrient content in soil and weather factors. The impact of the establishment method on filled grains per panicle proved in earlier study [49]. Higher filled grain per panicle was contingent upon vigorous tiller growth with the photosynthates production from flag leave [50]. On the other hand, relatively small sink capacity or deficient source content and export presumably responsible for lower number of filled grains per panicle in DSR [51]. Excess seeding for getting more panicles per $\mathrm{m}^{2}$ and suppressing weeds [52] is often accompanied by reduced panicle size, 1000 grain weight and higher spikelet sterility.

\section{Yield and duration}

Ample yield was gained from treatment $V_{4} \times T_{1}(4.42 \mathrm{t} / \mathrm{ha})$ followed by $\mathrm{V}_{2} \times \mathrm{T}_{3}(4.34 \mathrm{t} / \mathrm{ha})$. The lowest was yielded by treatments $\mathrm{V}_{3} \times \mathrm{T}_{2}(2.70 \mathrm{t} / \mathrm{ha})$ followed by $\mathrm{V}_{3} \times \mathrm{T}_{1}(2.72 \mathrm{t} / \mathrm{ha})$. Straw yield was most in $\mathrm{V}_{3} \times \mathrm{T}_{1}(5.10 \mathrm{t} / \mathrm{ha})$ and least in treatment $\mathrm{V}_{1} \times \mathrm{T}_{3}(2.72 \mathrm{t} / \mathrm{ha})$. In case of duration, treatment $\mathrm{V}_{4} \times \mathrm{T}_{3}$ took maximum days (111.67 days); contrary treatment $\mathrm{V}_{1} \times \mathrm{T}_{2}$ was harvested at 96.83 days (Figure 8). Here, more straw yield resulted less grain yield and vice versa; but Binadhan-21 with $120 \%$ and BRRI dhan85 with $80 \%$ recommended fertilizer dose performed better. Chowhan., et al. [44] found mean grain, straw and duration of Binadhan-21 under dibbling seeded was found $4.30 \mathrm{t} / \mathrm{ha}, 4.80 \mathrm{t} / \mathrm{ha}$ and 101 days. In TPR Chowhan., et al. [22] reported grain, straw and field duration of Binadhan-19 was 4.37 t/ha, 5.05 t/ha and 99.33 days. Deviation of yield and duration might be for difference in crop establishment method.

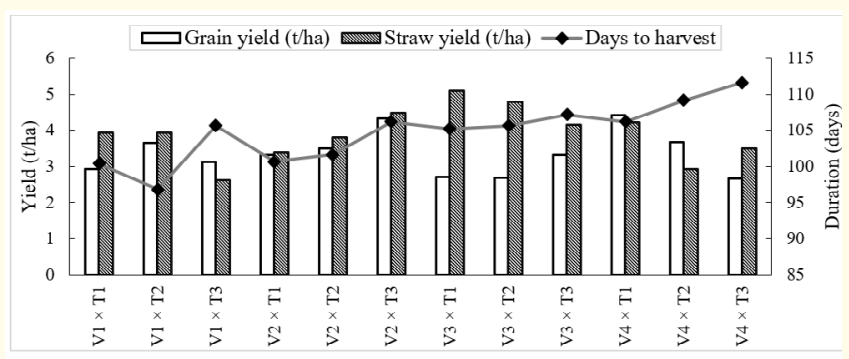

Figure 8: Yield and maturity duration influenced by variety and fertilizer dose.

In terms of variety-soaking interaction, treatment $\mathrm{V}_{4} \times \mathrm{S}_{0}$ yielded most (4.09 t/ha) followed by $\mathrm{V}_{4} \times \mathrm{S}_{0}(3.84 \mathrm{t} / \mathrm{ha})$. Minimum and alike yield was obtained in $V_{3} \times S_{24}(3.84 \mathrm{t} / \mathrm{ha})$ and $\mathrm{V}_{3} \times \mathrm{S}_{0}(3.01 \mathrm{t} / \mathrm{ha})$. Straw yield was highest in $V_{3} \times S_{0}(4.93 \mathrm{t} / \mathrm{ha})$ and the lowest (3.51 $\mathrm{t} / \mathrm{ha}$ ) was obtained from treatments $\mathrm{V}_{1} \times \mathrm{S}_{0}, \mathrm{~V}_{1} \times \mathrm{S}_{24}$ and $\mathrm{V}_{4} \times \mathrm{S}_{24}$. Treatment $\mathrm{V}_{4} \times \mathrm{S}_{24}$ was harvested late (109.67 days) and $\mathrm{V}_{1} \times \mathrm{S}_{0}$ was harvested at the earliest (99.89 days) (Figure 8).

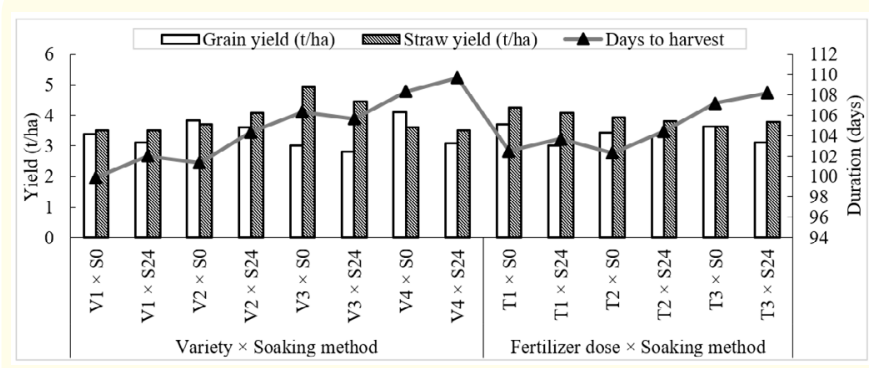

Figure 9: Yield and maturity of DSR as effected by variety, fertilizer dose and soaking methods.

Fertilizer dose-soaking effect exhibited, treatment $\mathrm{T}_{1} \times \mathrm{S}_{0}$ produced abundant yield (3.70 t/ha) and $\mathrm{T}_{1} \times \mathrm{S}_{24}$ least yield (3.01 t/ ha) among the treatments. Straw yield was non-significant hence no effect was spotted. But maturity duration was significantly influenced and maximum was found with treatment $\mathrm{T}_{3} \times \mathrm{S}_{24}(108.17$ days) and the minimum was seen with $\mathrm{T}_{2} \times \mathrm{S}_{0}$ (102.25 days) followed by $\mathrm{T}_{1} \times \mathrm{S}_{0}$ (102.50 days) treatment (Figure 9). Best yield was obtained without seed priming. This may have occurred for enough soil moisture and favorable rapid germination factors. Duration of crop was more or less alike according to BRRI [42] and Chowhan., et al. [22]. 


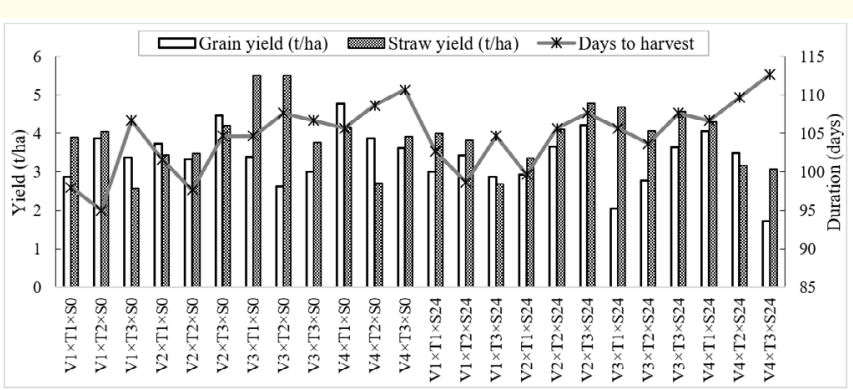

Figure 10: Interaction of variety, fertilizer dose and soaking method on yield and maturity of DSR.

Interaction effect of variety-fertilizer-soaking method alluded that, utmost grain yielded from treatment combination $\mathrm{V}_{4} \times \mathrm{T}_{1} \times \mathrm{S}_{0}$ (4.78 t/ha) followed by $\mathrm{V}_{2} \times \mathrm{T}_{3} \times \mathrm{S}_{0}(4.46 \mathrm{t} / \mathrm{ha})$. Minimal grain yielded from $\mathrm{V}_{4} \times \mathrm{T}_{3} \times \mathrm{S}_{24}(1.72 \mathrm{t} / \mathrm{ha})$. Statistically identical and maximal straw yield was noted with treatment $V_{3} \times T_{1} \times S_{0}(5.52 t / h a)$ followed by $\mathrm{V}_{3} \times \mathrm{T}_{2} \times \mathrm{S}_{0}(5.52 \mathrm{t} / \mathrm{ha})$ and lowest was found with $\mathrm{V}_{1} \times \mathrm{T}_{3}$ $\times \mathrm{S}_{0}(2.57 \mathrm{t} / \mathrm{ha})$ treatment. Longest days to harvest was needed in $\mathrm{V}_{4} \times \mathrm{T}_{3} \times \mathrm{S}_{24}\left(112.67\right.$ days); while $\mathrm{V}_{1} \times \mathrm{T}_{2} \times \mathrm{S}_{0}$ was harvested at shortest (95.00 days) period (Figure 10). Abbas., et al [53] concluded fertilization technique influenced yield, yield attributes and nutrient uptake in DSR. Ghosh., et al. [54] compared fifteen aus rice varieties under irrigated and rainfed state and mentioned, grain yield under rainfed condition reduced between $3.59 \%$ to $17.06 \%$ and a reduction of harvest index ranged from $0.26 \%$ to $4.71 \%$.
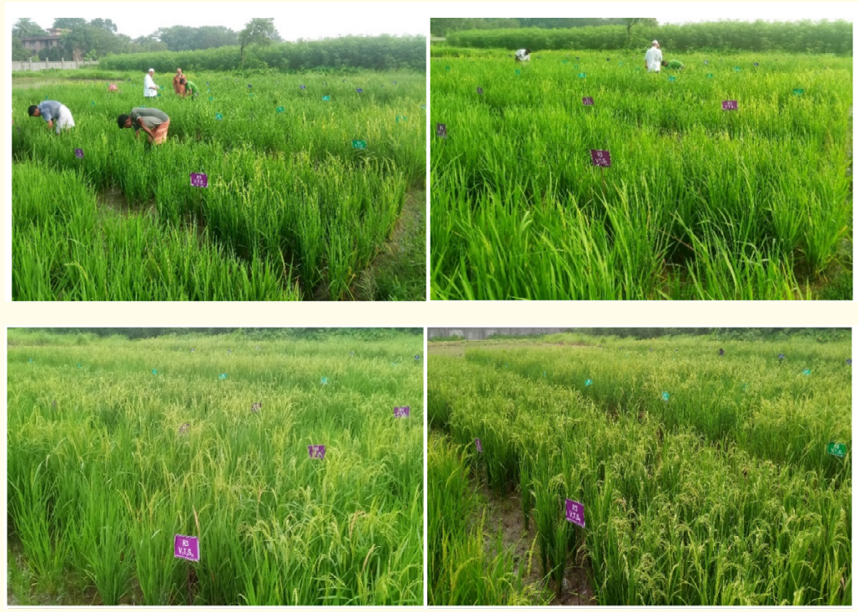
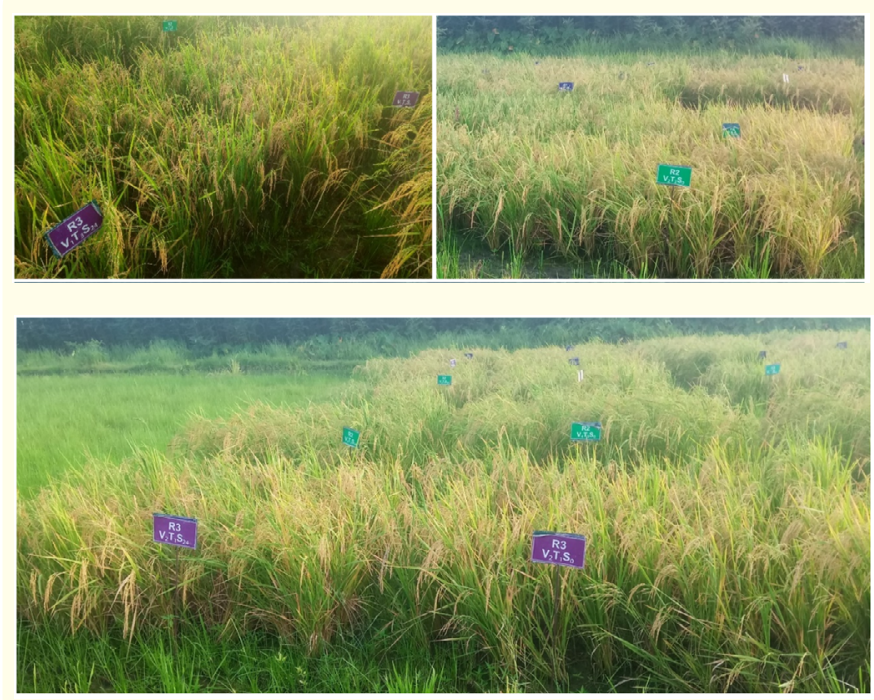

Figure 11: Treatment plots of aus rice varieties at early flowering, grain filling and harvesting stage.

\section{Conclusions}

Investigation results suggest that BRRI dhan85 and Binadhan-21 with no hydro soaking along with $80 \%$ recommended fertilizer dose gave optimum grain and straw yield. But, Binadhan-21 was more fertilizer responsive than BRRI dhan85 in terms of biological yield. Overall, it was remarkably noticed that application of more fertilizers resulted increased grain sterility, vegetative growth and maturity duration. As this trial covered a limited time and area further trials are necessary to justify the role of different fertilizer doses on the yield and related attributes under dry-DSR system.

\section{Acknowledgements}

Authors would like to express sincere appreciativeness to Scientific Officers Khan Jahan Ali and Moshiur Rahman. Authors also like to convey heartfelt thanks to Abdus Sobhan, Farm Manager, Julekha Khatun, Field Man, all staffs specially, Isaq, Selim, Mamun, Arif and labors specially Akram, Ujjal and Monsur of BINA Sub-station, Ishurdi, Pabna for their helpful coordination during the entire experiment work. We profoundly acknowledge the cooperation of Sharif Ahmed, PhD, senior specialist-Agronomy, CSISA phase III, International Rice Reseearch Institute regarding his timely suggestion, advice, direction and solution on various aspect of the field 
experimentation. We are thankful to Mr. Md. Mahbubur Rahman Dewan, Senior Scientific Officer of Bangladesh Rice Research Institute, Regional station, Kushtia for providing seeds for this special trial. Overall we are grateful to the Bangladesh Institute of Nuclear Agriculture for providing necessary supports to conduct this research investigation.

\section{Disclaimer}

A portion of this experimental findings (as abstract) were presented at "International Conference on Renewable Energy, Sustainable Environmental and Agricultural Technologies (i-RESEAT 2021) Maejo University, Chiang Mai, Thailand, December 22-23, 2021.

\section{Bibliography}

1. Anum W., et al. "Irrigation water status in pakistan, challenges and management strategies: a mini review". Journal of Quality Assurance in Agricultural Sciences 1.1 (2021): 14-21.

2. Chowhan S. "Impact of Agro Technology on Socio-economic Condition of the Farming Groups at Jagannathpur". International Journal of Agriculture, Environment and Biotechnology 14.1 (2021): 97-109.

3. Nagarjuna P., et al. "Effect of Crop Establishment Methods and Nitrogen Management on Growth and Yield of Rice". International Journal of Agriculture, Environment and Biotechnology 14.2 (2021): 185-189.

4. Bista B. "Direct Seeded Rice: A New Technology for Enhanced Resource-Use Efficiency". International Journal of Applied Sciences and Biotechnology 6.3 (2018): 181-198.

5. Pandey S., et al. "Direct seeding: research issues and opportunities". Proceedings of the International Workshop on Direct Seeding in Asian Rice Systems: Strategic Research Issues and Opportunities, 25-28 January 2000, Bangkok, Thailand. Los Baños (Philippines): International Rice Research Institute (2002): 383.

6. Malla Razan. "East African Scholars Journal of Agriculture and Life Sciences 4.3 (2021): 75-81.

7. Kumar SG., et al. "Effect of non-conventional system of cultivation with varied $\mathrm{N}$ levels on growth, yield and water management of rice (Oryza sativa L.)". Madras Agricultural Journal 95.7-12 (2008): 343-352.
8. Bhattacharyya R., et al. "Effect of tillage and irrigation on yield and soil properties under rice (Oryza sativa) - wheat (Triticum aestivum) system on a sandy clay loam soil of Uttaranchal". Indian Journal of Agricultural Sciences 76.7 (2006): 405-409.

9. Wassmann R., et al. "Mitigating greenhouse gas emissions from rice-wheat cropping systems in Asia". Environment, Development and Sustainability 6 (2004): 65-90.

10. Balasubramanian V and Hill JE. "Direct wet seeding of rice in Asia. Emergence issues and strategic research needs for the 21st century". Direct seeding: research strategies and opportunities. Proceedings (2002): 15-39.

11. Bhushan L., et al. "Saving of water and labor in a rice-wheat system with no-tillage and direct seeding technologies". Agronomy Journal 99.5 (2007): 1288-1296.

12. Khade VN., et al. "Effect of seeding rates and level of $\mathrm{N}$ on yield of direct-seeded (Rahu) summer rice in Konkan". Journal of Maharashtra agricultural University 18 (1993): 32-35.

13. Sattar MA and SI Bhuiyan. "Performance of Direct-Seeded and Transplanted Rice under Different Water Management Practices". Bangladesh Rice Journal 5.1-2 (1994): 1-5.

14. Singh S., et al. "Performance of Direct-Seeded Rice as Influenced by Dual Cropping with Nitrogen Levels and Weed Management Practices in Prayagraj Region of Eastern Uttar Pradesh". National Academy Science Letters 43 (2020): 399402.

15. Mohanta S., et al. "Productivity and Profitability of Kharif Rice Are Influenced by Crop Establishment Methods and Nitrogen Management in the Lateritic Belt of the Subtropical Region". Agronomy 11 (2021): 1280.

16. Cornell University. SRI-RICE, SRI International Network and Resources Center (2020).

17. Midya A., et al. "Crop Establishment Methods and Integrated Nutrient Management Improve: Part II. Nutrient Uptake and Use Efficiency and Soil Health in Rice (Oryza sativa L.) Field in the Lower Indo-Gangetic Plain, India". Agronomy 11 (2021)1894.

18. Joshi Ekta., et al. "Management of direct seeded rice for enhanced resource - use efficiency". Plant Knowledge Journal 2.3 (2013): 119-134.

19. FAOSTAT 2021. Crops and livestock products (2021). https:// www.fao.org/faostat/en/\#data/QCL 
20. BBS (Bangladesh Bureau of Statistics). Yearbook of Agricultural Statistics-2020. 32nd Series, Planning Division, Ministry of Planning, Dhaka, Bangladesh (2020): 1-51.

21. BARC. "Suitability Map of Aus [Pabna: Ishwardi] (2021).

22. Chowhan S., et al. "Yield and morpho physical characters of some modern aus rice varieties at Khagrachari". Plant Science Today 8.1 (2021b): 155-160.

23. Singh SP., et al. "Direct Seeded Rice: Prospects, Constraints, Opportunities and Strategies for Aerobic Rice (Oryza sativa L) in Chhattisgarh - A Review". Int. J. Curr. Microbiol. App. Sci 8.09 (2019): 889-912.

24. Gao XP., et al. "From flooded to aerobic conditions in rice cultivation: consequences for zinc uptake". Plant Soil 280 (2006): 41-47.

25. Chowhan S and Islam M. "Zinc Effects Yield of Mustard (Brassica campestris L.) Under Zero Tillage". Asian Journal of Soil Science and Plant Nutrition 8.1 (2021): 27-35. https://doi. org/10.9734/ajsspn/2021/v8i130126

26. PRC. "Weather data of 2020. Pulses Research Center". Bangladesh agricultural research institute, Ishurdi, Pabna-6620, Bangladesh (2021).

27. FRG. "Fertilization Recommendation Guide". Bangladesh Agricultural Research Council (BARC), Farmgate, Dhaka 1215 (2012): 01-258.

28. Ahmmed S., et al. "Fertilizer recommendation guide-2018". Dhaka Bangladesh Agricultural Research Council (2018): 1221.

29. Ahmed S. "Crop and Weed Management Strategies for Dry Direct Seeded Rice”. Eliva Press (2020): 143.

30. Mahbub MM and MKA Bhuiyan. "Performance of Bensulfuran methyl $12 \%$ + Bispyribac sodium 18\% WP against annual weeds in transplanted rice (Oryza sativa) cultivation in Bangladesh". Scientia Agriculturae 21.3 (2018): 85-92.

31. DAE (Department of Agricultural Extension). List of Registered Agricultural Pesticides, Bio Pesticides and Public Health Pesticides in Bangladesh (2021).

32. Chowhan S., et al. "Comparative on farm performance of five modern rice varieties with two local cultivars". Journal of Bioscience and Agriculture Research 13.01 (2017): 1074-1086.
33. Ahmed Sharif., et al. "Optimum sowing date and cultivar duration of dry-seeded boro on the High Ganges River Floodplain of Bangladesh". Field Crops Research 190 (2016): 91-102.

34. Ahmed Sharif and Bhagirath Singh Chauhan. "Performance of Different Herbicides in Dry-Seeded Rice in Bangladesh". The Scientific World Journal 2014 (2014): 14.

35. Statistix. "Data analysis software for researchers (Version 10.0)". Analytical Software, 2105 Miller Landing Rd, Tallahassee Florida 32312, USA (2021).

36. Russell DF MSTAT-C computer package programme. Crop and Soil Sci Dept., Michigan State University., US (1986).

37. Gomez KA and Gomez AA. "Statistical producers for agricultural research. A Wiley Int. Sci. Pub. John Wiley and Sons, New York, Brisbane, Singapore (1984): 139-240.

38. Yasumoto., et al. "Changes in developmental duration of direct-seeded rice in a well-drained paddy field in response to late planting". Plant Production Science 20.3 (2017)279-287.

39. Kaur Simerjeet and Surjit Singh. "Influence of plant densities on growth and yield of direct seeded rice (Oryza sativa L.)". Agricultural Research Journal 53.4 (2016): 594-596.

40. Ghosh SR., et al. "Adjusting Planting Time of Binadhan-17 in Boro Season". Journal of Phytology 13 (2021): 21-27.

41. BRRI (Bangladesh Rice Research Institute). BRRI dhan82 leaflet (In Bengali) (2017A).

42. BRRI (Bangladesh Rice Research Institute). BRRI dhan85 leaflet (In Bengali) (2017B).

43. BINA (Bangladesh Institute of Nuclear Agriculture). (2020). "Annual Report of Bangladesh Institute of Nuclear Agriculture 2019-20". Mymensingh-2202, Bangladesh (2020): 10-260.

44. Chowhan S., et al. "Evaluation of NERICA rice mutant in Jhum cultivation". International Journal of Agronomy and Agricultural Research 12.2 (2018): 24-31.

45. Jnanesha AC and Ashish Kumar. "Effect of Crop Establishment Methods on Growth Yield and Water Productivity of Rice". International Journal on Agricultural Sciences 8.1 (2017): 40-45.

46. Xu Le., et al. "Comparing the Grain Yields of Direct-Seeded and Transplanted Rice: A Meta-Analysis" Agronomy 9.11 (2019): 767. 
47. Mahajan G., et al. "Seed Priming Effects on Germination, Growth and Yield of Dry Direct-Seeded Rice". Journal of Crop Improvement 25.4 (2011): 409-417.

48. BRKB (Bangladesh Rice Knowledge Bank). Problems and solution of rice production (2019): 23.

49. Ramesh S and Chandrasekaran B. "Evaluation of crop establishment methods and nitrogen management strategies on realizing yield potential of rice hybrid ADTRH 1". Asian Journal of Plant Sciences 6.2 (2007): 239-251.

50. Zhu D., et al. "Effects of nitrogen level on yield and quality of japonica soft super rice". Journal of Integrative Agriculture 16.5 (2017): 1018-1027.

51. Yang J and Zhang J. "Crop management techniques to enhance harvest index in rice”. Journal of Experimental Botany 61.12 (2010): 3177-3189.

52. Chauhan BS and Johnson DE. "Row spacing and weed control timing affect yield of aerobic rice". Field Crops Research 121.2 (2011): 226-231.

53. Abbas RN., et al. "Weed-Free Durations and Fertilization Regimes Boost Nutrient Uptake and Paddy Yield of Direct-Seeded Fine Rice (Oryza sativa L.)”. Agronomy 11.12 (2021): 2448.

54. Ghosh U., et al. "Yield Response of Direct Seeded Aus Rice Varieties under Rainfed Condition". American Journal of Plant Sciences 9 (2018): 416-434.

\section{Assets from publication with us}

- Prompt Acknowledgement after receiving the article

- Thorough Double blinded peer review

- Rapid Publication

- Issue of Publication Certificate

- High visibility of your Published work

Website: www.actascientific.com/

Submit Article: www.actascientific.com/submission.php

Email us: editor@actascientific.com

Contact us: +919182824667

Citation: Sushan Chowhan and Kamrun Nahar. "Evaluating the Role of Fertilizer and Seed Soaking on Direct Seeded Aus Rice Varieties". Acta Scientific Agriculture 6.2 (2022): 02-17. 